\title{
Non-Rayleigh Scattering by a Randomly Oriented Elongated Scatterer
}

by

Saurav Bhatia

B.S., Cornell University (2010)

Submitted to the Department of Electrical Engineering and Computer

Science

in partial fulfillment of the requirements for the degree of

Master of Science in Electrical Engineering and Computer Science

$$
\begin{gathered}
\text { at the } \\
\text { MASSACHUSETTS INSTITUTE OF TECHNOLOGY } \\
\text { and the }
\end{gathered}
$$

BOATVES

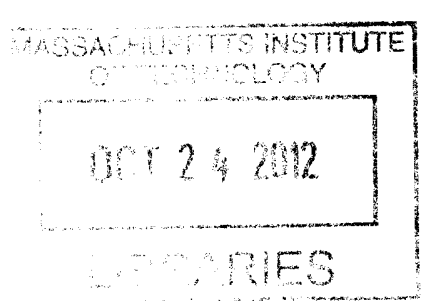

September 2012

(C) Saurav Bhatia, MMXII. All rights reserved.

The author hereby grants to MIT and WHOI permission to reproduce and distribute publicly paper and electronic copies of this thesis document in whole or in part.

Author ...........

Department of Electrical Engineering and Computer Science

Certified by . -

-

August 30, 2012

Timothy K. Stanton

Senior Scientist A.D . . . . Thesis Supervisor

Accepted by .....

Chair, Department Comentitee (Leslie A. Kolodziejski Accepted by ...... 



\title{
Non-Rayleigh Scattering by a Randomly Oriented Elongated
}

\section{Scatterer}

by

\author{
Saurav Bhatia \\ Submitted to the Department of Electrical Engineering and Computer Science \\ on August 30, 2012, in partial fulfillment of the \\ requirements for the degree of \\ Master of Science in Computer Science and Engineering
}

\begin{abstract}
The echo statistics of a randomly rough, randomly oriented prolate spheroid that is randomly located in a beampattern are investigated from physics-based principles both analytically and by Monte Carlo methods. This is a direct-path geometry in which reflections from neighboring boundaries are not a factor. The center of the prolate spheroid is assumed to be confined to the plane containing the MRA (maximum response axis). Additionally, the rotation of the prolate spheroid is assumed to always be in this plane. The statistics and, in particular, the tails of the probability density function (PDF) and probability of false alarm (PFA) are shown to be strongly non-Rayleigh and a strong function of shape of scatterer. The tails are shown to increase above that associated with a Rayleigh distribution with increasing degree of elongation (aspect ratio) of the scatterer and when roughness effects are introduced. And, as also shown in previous studies, the effects associated with the scatterer being randomly located in the beam contribute to the non-Rayleigh nature of the echo. The analytically obtained results are compared to Monte Carlo simulations for verification.
\end{abstract}

Thesis Supervisor: Timothy K. Stanton Title: Senior Scientist 


\section{Acknowledgments}

I would like to thank my research advisor, Dr. Timothy K. Stanton from Woods Hole Oceanographic Institution, for his insight and guidance throughout the course of this project. Dr. Stanton's advice and support was extremely important and helpful to me, from the time when I first started work on this project up until the final copies were printed. The discussions I had with the members of our research group, Kyungmin Baik and Wu-Jung Lee, were also instrumental in helping me bring this project to a successful conclusion.

I am grateful to the Academic Programs Office at WHOI and the Office of Naval Research for their support, both financial and administrative, without which this project would not have been possible. This research was funded, in part, by the U.S. Office of Naval Research grant nos. N00014-11-1-0116 and N00014-10-1-0127.

Finally, I would like to thank my friends and family for their unconditional love and support throughout the course of this project. 


\section{Contents}

1 Introduction $\quad 10$

2 Overview of general scattering and statistical formulations $\quad 15$

2.1 Elementary formulas concerning random variables . . . . . . . . 17

2.1.1 Distribution of a function of a random variable . . . . . 17

2.1.2 Distribution of the product of two random variables . . . . . 19

2.1.3 Derivation of the Rayleigh PDF . . . . . . . . . . 21

3 Beampattern Effects $\quad 23$

3.1 General Theory . . . . . . . . . . . . . . . . . 23

3.2 Applications to Circular Aperture . . . . . . . . . . . . . 26

4 Scattering by Prolate Spheroids (No Beampattern Effects) 31

4.1 Deterministic Scattering (Fixed Orientation, Smooth Spheroid) . . . . 32

4.2 Stochastic Effects (Random Orientation, Smooth Spheroid) . . . . . . 34

4.3 Stochastic Effects (Random Orientation, Rough Spheroid) . . . . . 36

5 Scattering by Prolate Spheroids with Beampattern Effects $\quad 40$

6 Monte Carlo Validation and Computational Issues $\quad 44$

6.1 Monte Carlo Validation . . . . . . . . . . . . . . . . . 45

6.2 Computational Issues . . . . . . . . . . . . . . . . . . 46 
7 Summary and Conclusion

A Matlab Code $\quad 53$

A.1 ellipsoid.m ..................... 53

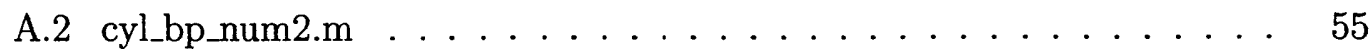

A.3 prosph_simulation.m . . . . . . . . . . . 57

A.4 pdfmultiplier $2 . \mathrm{m} \ldots \ldots \ldots \ldots \ldots \ldots \ldots$

A.5 roughprolatespheroid.m. . . . . . . . . . . . 59 


\section{List of Figures}

1-1 Diagram illustrating the scattering geometry of the prolate spheroid treated in this thesis. . . . . . . . . . . . . . 11

3-1 The amplitude of a two-way composite beampattern (3.7) for a circular aperture with $k a=2 \pi$. This corresponds to a beamwidth of approximately $30^{\circ}$. The points of interest marked A, B and C indicate regions where the slope of the curve is zero. These points correspond to the singularities labeled in Fig. 3-2. . . . . . . . . . . . . . .

3-2 The PDF of the two-way composite beampattern amplitude for a circular aperture with $k a=2 \pi$. The scatterer is assumed to be limited to the MRA plane, i.e. $p_{\theta}=$ constant. The PDF for narrower beams is qualitatively similar, as shown in Fig. 3-3. The singularities marked $\mathrm{A}, \mathrm{B}$ and $\mathrm{C}$ correspond to points of zero slope in the beampattern amplitude curve (Fig. 3-1) . . . . . . . . . . . . . . 28

3-3 The two-way composite beampattern for a circular aperture with $\mathrm{ka}=$ $2 \pi$. In contrast to Fig. $3-2$, the scatterer is assumed to lie in the halfspace, i.e. $p_{\theta}=\sin (\theta)$. Note the absence of a singularity corresponding

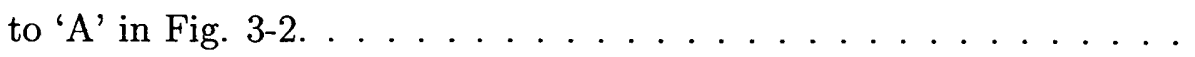


3-4 The two-way composite beampatterns for circular apertures with various values of $\mathrm{ka}$, demonstrating the increase in the number of singularities due to additional sidelobes. The center of the prolate spheroid is assumed to be confined to the MRA plane. . . . . . . . . . . 30

4-1 Scattering amplitude with varying angle of incidence for prolate spheroids of constant volume and different aspect ratios. . . . . . . . . . . . 34

4-2 PDFs of scattering amplitude for smooth prolate spheroids that are randomly and uniformly oriented in a plane that also contains the incident field. Beampattern effects are not included. . . . . . . . . 36

4-3 PDFs of scattering amplitude for randomly oriented rough prolate spheroids of varying aspect ratios, without beampattern effects. The circular markers represent the results of Monte Carlo simulations for validation, discussed later in the thesis. . . . . . . . . . .

5-1 Scattering amplitude PDFs for various scatterers, with beampattern

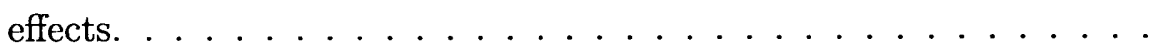

5-2 Echo amplitude distributions for rough prolate spheroids of varying aspect ratios, with beampattern effects. The data points are the results of Monte Carlo simulations for validation, discussed later in the paper. The plot on the right provides a zoomed-in view focusing on the tails of the PDFs for prolate spheroids of various aspect ratios. . . . . .

5-3 Plot of the scattering amplitude PFAs for various types of scatterers (corresponding to Fig. 5-1), as well as a plot focused on the tails of

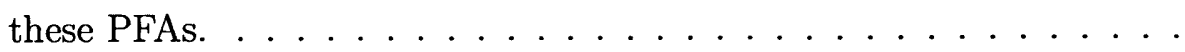


6-1 Beampattern PDF associated with two-way composite beampattern for a circular aperture with $\mathrm{ka}=2 \pi$. The numerically calculated curve (solid line) based on analytical formulas (3.1) and (3.7), is compared to a Monte Carlo simulation of a scatterer with constant scattering amplitude in the beam (circles). The simulation data is divided into bins with equal width on a $\log$ scale. . . . . . . . . . .

6-2 Beampattern PDF associated with two-way composite beampattern for a circular aperture with $\mathrm{ka}=2 \pi$, using (3.1) and (3.7). The numerically calculated curve (solid line) is compared to a Monte Carlo simulation of a scatterer with constant scattering amplitude in the beam (circles). The simulation data is adaptively divided into bins with varying width. The width of bins is much lower near the singularities present in the

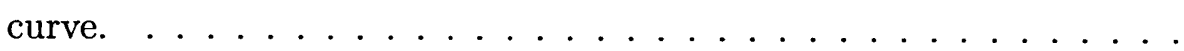




\section{Chapter 1}

\section{Introduction}

Active sonar and radar systems are an important means of remote sensing in the environment. Such systems use transducers to modulate and direct waves into their surroundings. By detecting the scattered signal, remote sensing systems can infer information about the scatterer or its environment. In typical use, sonar and radar systems need to process data about objects of interest while filtering data from unwanted sources. Clutter is defined as a signal that closely resembles the target signature but is caused by objects that are not of interest.

If the number of targets within the resolution of a remote sensing system is large, the distribution of the scattered signal tends towards a Gaussian distribution due to the central limit theorem. As a result, the envelope of the echo follows a Rayleigh distribution. In this case, the probability of false alarm (PFA) decays at an exponential rate with increasing echo amplitude. If there are comparatively fewer scatterers relative to the system's resolution, the probability distribution function (PDF) of the envelop may be non-Rayleigh. In such a case, the tail of the non-Rayleigh PDF is typically not bounded by an exponential decay. Such distributions are known as heavy tailed distributions and lead to an increase in the PFA of the system $[1,2]$. The increase in false alarm signals implies that characterization and modeling of clutter 
is very important for accurate remote sensing.

In a purely stochastic approach to modeling the echo PDF of clutter, standard heavy tailed probability distributions like the Weibull or the K-distribution are fitted to empirically obtained amplitude data. The correlation between the parameters of the theoretical distribution and the physical characteristics of clutter is then inferred statistically, rather than being derived from first principles. The majority of previous studies on modeling radar and sonar clutter utilize a similar approach [1-4]. A statistical model for the echo amplitude of clutter must account for several factors that will affect the shape of the distribution and the degree to which it is non-Rayleigh [5]. Physical characteristics of the scatterers such as orientation with respect to the incident wave and surface roughness will affect the shape of the distribution.

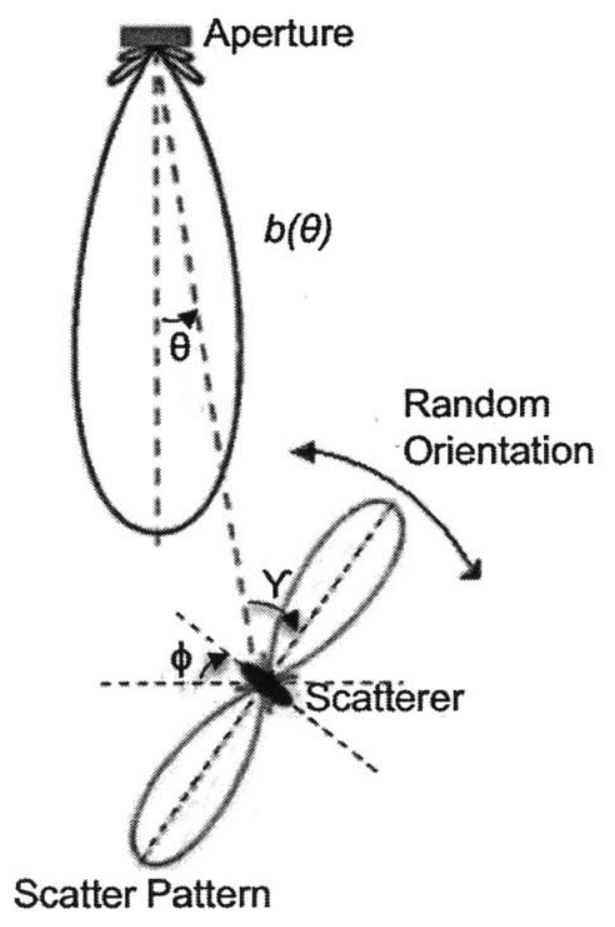

Figure 1-1: Diagram illustrating the scattering geometry of the prolate spheroid treated in this thesis. 
With the assumption that some physical characteristics of the sources of clutter are known, the echo amplitude PDF can be derived using physics-based principles. Since many features in nature are elongated, this thesis aims to derive the probability distribution of the echo amplitude of a prolate spheroid with a random location, orientation, and roughness. Because of the many complexities of this problem, the predictions are limited to the following conditions: 1) the scattering is calculated in the high frequency geometric optics limit in which the wavelength of the incident field is much smaller than any dimension of the object, 2) all motion- changes in location and orientation- is in the same plane that contains the maximum response axis (MRA) of the beam as illustrated (Fig. 1-1), and 3) only direct path scattering is considered in which scattering by neighboring boundaries is not a factor.

The physics-based approach to deriving the PDF of the echo amplitude begins with the deterministic echo amplitude for a prolate spheroid at an orientation $\phi$ with respect to the horizontal axis (Fig. 1-1). The center of the prolate spheroid is located in the plane of the MRA of the transducer and its azimuthal coordinate is given by the angle $\theta$. The angle $\gamma$ is formed between the perpendicular axis of the prolate spheroid and the line connecting its center to the transducer. The polar axis of the spheroid lies entirely within the MRA plane and the axis of rotation is perpendicular to that plane. One real world scenario that corresponds to these conditions is the case of a sidescan sonar that is insonifying fish at the same depth.

The problem of finding the deterministic scattering amplitude of a scatterer of known shape at a fixed location in the beampattern is well studied in literature. Bowman et al. [6] have written a text that analytically derives the scattering amplitudes of infinite, semi-infinite and finite 2- and 3-dimensional bodies with a variety of different shapes, including the prolate spheroid case considered here. In this paper, $\phi$ is a random variable with a known PDF. The echo amplitude of the prolate spheroid is then a function of the random variable $\phi$. Fundamental probability theory can be 
used to derive the PDF for the scattering amplitude of a randomly oriented object, when the probability distribution of $\phi$ is already known [3]. A similar approach has been used in the past to derive the echo PDF of a patch of scatterers with arbitrary echo distributions in the beam $[7,8]$. The effect of the randomly rough surface of the spheroid is then included heuristically by multiplying the scattering amplitude with a Rayleigh distributed random variable $[9,10]$.

As the location of the scatterer in the beam is a random variable, the effect of the transducer's beampattern on the echo amplitude is correspondingly random. Therefore, another change of variables operation is required to find the PDF of the beampattern of the transducer. The echo amplitude as "seen" through the receiver for a prolate spheroid with random location, orientation and roughness is the product of three random variables, where each variable is derived from one of the three physical parameters of the spheroid. The distribution of the product of random variables can be derived from elementary probability theory [11]. This formulation is used to find the final echo amplitude PDF for the rough prolate spheroid in the beam. In contrast to the statistical approach as cited above (i.e., using Weibull distributions, etc.), the derived PDF is explicitly dependent on the physical characteristics of the scatterer and the beampattern.

In this thesis, Chapter 2 provides an overview of the theory and statistical formulas that are important to deriving the echo amplitude PDF of a rough prolate spheroid in the beam. The derivations of well known equations that are critical to this research are also presented here, such as the distribution of a function of a random variable. In the following chapter, these formulations are used to derive the PDF of the echo amplitude of a two-way beampattern for the specific case of a circular aperture.

The PDF of the scattering amplitude of a randomly oriented prolate spheroid is then determined in Chapter 4. Initially, the deterministic scattering amplitude for a smooth prolate spheroid at a fixed orientation is presented. The orientation of 
the spheroid is then assumed to be random, and a PDF of the scattering amplitude is derived. Finally, the effect of surface roughness is accounted for in a heuristic manner, wherein the scattering amplitude is multiplied by a Rayleigh distributed random variable.

The formulas presented in Chapters 3 and 4 are then combined in Chapter 5 to find the echo amplitude PDF for a randomly rough, randomly oriented prolate spheroid that is randomly located in the beam. Chapter 6 provides an overview of the methods used to validate the analytical results presented in earlier chapters. In addition, the computational issues that were encountered while calculating the PDFs are described, and the solutions implemented are discussed. 


\section{Chapter 2}

\section{Overview of general scattering and statistical formulations}

The echo statistics will be formulated explicitly in terms of the physics of the scattering by the individual scatterer (prolate spheroid) and system parameters. This physics-based approach involves a sequence of steps, including describing the deterministic scattering by an arbitrarily oriented prolate spheroid that is arbitrarily located in the beam, and then randomizing the parameters, which results in a randomized echo. For a general scattering problem, the scattered pressure wave $P_{s}$ in phasor notation is dependent on the incident pressure $P_{0}$ and the scattering amplitude $f_{s}$ of the scatterer:

$$
P_{s}=P_{0} \frac{e^{i k r}}{r} f_{s}
$$

where $k$ is the wavenumber of the incident wave in the surrounding medium, and $r$ represents the range of the scatterer in spherical coordinates. This expression does not include system parameters, such as the beampattern. When modeling the complete system, beampattern effects must also be taken into account and the final received echo amplitude is:

$$
\tilde{e}=A b\left|f_{s}\right|
$$


where $A$ represents all system constants, $b$ is the two-way beampattern amplitude and $f_{s}$ is the scattering amplitude.

Since the scatterer is randomly located in the beam at angle $\theta$ and the beampattern is a function of the random variable $\theta$, then the beampattern can be treated as a random variable. With the echo from the scatterer (before beampattern effects) also being a random variable, then the echo $\tilde{e}$ received by the system (including beampattern effects) is the product of the two random variables, the beampattern $b$ and echo $\left|f_{s}\right|$ before beampattern effects. We use Ehrenberg's approach of describing this product, which involves directly applying classic formulas of statistical theory $[8,12]$.

In this high frequency limit, the scattering by a smooth prolate spheroid is calculated through use of the Kirchhoff approximation. The scattering amplitude is dependent on the angle $\gamma$ of the spheroid with respect to the incident wave. $\gamma$ can be derived from the randomly distributed angles $\theta$ and $\phi$. Since $\gamma$ is random, the scattering amplitude $f_{s}$ is also a random variable. Roughness is later introduced heuristically by modulating the deterministic scattering at a fixed orientation angle with a Rayleigh PDF. This is based on the fact that rough bounded objects have resulted in Rayleigh-distributed echoes at high frequencies $[9,10]$. Beampattern effects are incorporated through direct use of the formulation first derived by Ehrenberg in which the beam is considered to be a random variable due to the fact that the scatterer is randomly located in the beam [12].

In the below analysis, relevant elementary formulas from probability theory are first derived. The beampattern PDF from a circular aperture is then reviewed, and the stochastic properties $\left(p_{s}\right)$ of the scatterer (specifically, of a randomly rough, randomly oriented prolate spheroid) before beampattern effects are investigated. Finally, those two PDFs are combined through use of (2.12) to formulate the echo PDF of the prolate spheroid with beampattern effects. Because of the similarity in formulations, 
the general form of the integral in (2.12) is also used to describe the echo statistics from a randomly rough prolate spheroid before beampattern effects.

\subsection{Elementary formulas concerning random vari- ables}

This section reviews the derivations of several well known formulas that are important to this thesis. The distribution of a function of a random variable is required to derive the PDFs of the beampattern and scattering amplitude from the PDFs of the angles $\theta$ and $\gamma$.

Calculating the PDF of the product of random variables is necessary in two cases. Firstly, the statistical effects of roughness on echo amplitude are included heuristically, by multiplying the scattering amplitude with a Rayleigh distributed random variable. Secondly, the beampattern must be multiplied with the scattering amplitude in order to determine the echo amplitude of the system, as described in (2.2).

The analytical expression for the Rayleigh PDF is also derived from the magnitude of the vector sum of two zero-mean Gaussian random variables. The common natural occurrence of vectors that have components modeled by uncorrelated Gaussian distributions emphasizes the importance of the Rayleigh PDF. This equation is effective in providing a statistical description of phenomena as diverse as wave heights in the ocean and the peak power of received radio signals [13].

\subsubsection{Distribution of a function of a random variable}

In this thesis, the angles $\theta$ and $\phi$ are assumed to be random variables with PDFs that are known a priori. The functions describing the beampattern and scattering amplitude are dependent on $\theta$ and $\gamma$, respectively. A change of variables operation is required to derive the PDF of these functions from the known distributions of the 
angles. This is a well studied statistical problem, with broad applications to applied probability [14].

Let $X$ and $Y$ be two random variables such that $Y=g(X)$. Without loss of generality, let there be an event $E$ that is said to occur if $X$ and $Y$ lie within small intervals $d x$ and $d y$, respectively. Then, the probability of $X$ lying in an interval $d x$ must be equal to the probability of $Y$ lying in an interval $d y$. This property allows the derivation of the relationship between the PDFs of $X$ and $Y$ using the differential $d Y / d X=g^{\prime}(X)$. By the definition of a PDF:

$$
p_{Y}(y) d y=p_{X}(x) d x
$$

where $p_{X}$ and $p_{Y}$ are the PDFs of random variables $X$ and $Y$, respectively.

If $g(X)$ is not monotonic, then $p_{Y}(y) d y$ must equal the sum of all probabilities in $X$-space that correspond to the $n$ solutions of $Y=g(X)$ :

$$
p_{Y}(y) d y=\sum_{i=1}^{n} p_{X}\left(x_{i}\right) d x_{i}
$$

Now, $d x_{i}$ is rewritten as $d y / g^{\prime}\left(x_{i}\right)$ :

$$
p_{Y}(y) d y=\sum_{i=1}^{n} \frac{p_{X}\left(x_{i}\right) d y}{g^{\prime}\left(x_{i}\right)}
$$

Upon canceling $d y$ from both sides, we obtain the desired formulation for the PDF of the function of a random variable:

$$
p_{Y}(y)=\sum_{i=1}^{n} \frac{p_{X}\left(x_{i}\right)}{g^{\prime}\left(x_{i}\right)}
$$




\subsubsection{Distribution of the product of two random variables}

As shown in (2.2), the echo amplitude of a scatterer can be described as the product of two random variables, the beampattern $b$ and the scattering amplitude $\left|f_{s}\right|$. In this case, the beampattern and scattering amplitude are assumed to be random variables with known PDFs $p_{b}$ and $p_{s}$ respectively. In order to find the PDF of $\tilde{e}$ in terms of $p_{b}$ and $p_{s}$, the relation between the joint distributions $p_{\tilde{e}, s}$ and $p_{b, s}$ is first derived [15]. For clarity, the presence of system constants in the term $A$ in (2.2) is ignored. Due to normalization, the value of $A$ will have no bearing on the results presented in this thesis.

Let $\mathrm{X}$ and $\mathrm{Y}$ be vector random variables that are defined as follows:

$$
X=\left[\begin{array}{c}
b \\
\left|f_{s}\right|
\end{array}\right] ; Y=\left[\begin{array}{c}
\tilde{e} \\
\left|f_{s}\right|
\end{array}\right]
$$

Then, $p_{Y}$ and $p_{X}$ correspond to the two joint distributions defined above. The Jacobian of the transformation $X=H(Y)$ is:

$$
J=\left[\begin{array}{cc}
1 /\left|f_{s}\right| & -\tilde{e} /\left|f_{s}\right|^{2} \\
0 & 1
\end{array}\right]
$$

Using fundamental probability theory, the PDF of $Y$ is [14]:

$$
p_{Y}=\|J\| p_{X}(H(Y))
$$

Therefore:

$$
p_{\tilde{e}, s}=\frac{1}{\left|f_{s}\right|} p_{s, b}\left(\frac{\left|f_{s}\right|, \tilde{e}}{\left|f_{s}\right|}\right)
$$

The PDF of $\tilde{e}$ can now be derived from the joint distribution by integrating out 
the variable $\left|f_{s}\right|$ :

$$
p_{\tilde{e}}(\tilde{e})=\int_{-\infty}^{\infty} \frac{1}{\left|f_{s}\right|} p_{s, b}\left(\left|f_{s}\right|, \frac{\tilde{e}}{\left|f_{s}\right|}\right) d\left|f_{s}\right|
$$

The PDFs of the beampattern and scattering amplitude are independent. In addition, the value of the scattering amplitude $\left|f_{s}\right|$ can never be smaller than that of the echo amplitude $\tilde{e}$, as the maximum possible value of the beampattern is unity. Taking this into account, the final equation for the PDF of the echo amplitude is:

$$
p_{\bar{e}}(\tilde{e})=\int_{\tilde{e}}^{\infty} \frac{1}{\left|f_{s}\right|} p_{s}\left(\left|f_{s}\right|\right) p_{b}\left(\frac{\tilde{e}}{\left|f_{s}\right|}\right) d\left|f_{s}\right|
$$

Due to the complex analytical description of $p_{e}$ and the presence of $1 / \mathrm{x}$ as a function argument within the integral, finding an analytical solution for (2.12) is very difficult and it is normally evaluated numerically except for some limiting cases.

From the above derivation, it is evident that the joint distributions $p_{\tilde{e}, b}$ and $p_{s, b}$ could have been considered instead. The result would then be the commuted form of (2.12):

$$
p_{\tilde{e}}(\tilde{e})=\int_{\tilde{e} /\left|f_{s}\right|}^{\infty} \frac{1}{b} p_{b}(b) p_{s}\left(\frac{\tilde{e}}{b}\right) d b
$$

Both of the equations described above will yield an identical result for the PDF $p_{\tilde{e}}$ of the echo amplitude. This property is used for the purposes of numerical evaluation in Matlab, as described in Section 6.2.

This same derivation can be applied when the echo amplitude of a rough prolate spheroid is calculated heuristically. In this case, the scattering amplitude of the smooth spheroid without beampattern effects is multiplied with a Rayleigh distributed random variable. 


\subsubsection{Derivation of the Rayleigh PDF}

Let $Z=\sqrt{X^{2}+Y^{2}}$, where $X$ and $Y$ are zero-mean independent, identically distributed (IID) Gaussian random variables. In this section, the variable $Z$ is shown to be Rayleigh distributed using a change of variables operation that is similar to the method used in Section 2.1.1. Let $E$ be the event corresponding to the small area $d x d y$ in Cartesian coordinates. Then, the probability of obtaining the area $d x d y$ must equal the probability of area $z d z d \theta$ in polar coordinates. An analytical expression for the distribution of $Z$ can be derived from this property [13].

The PDF of $\mathrm{X}$ (as well as $\mathrm{Y}$ ) is the well-known Gaussian distribution for the case of a zero mean, and can be written as [14]:

$$
p_{X}(x)=\frac{1}{\sqrt{2 \pi \sigma^{2}}} e^{-x^{2} / 2 \sigma^{2}}
$$

Since $X$ and $Y$ are independent by definition, their joint PDF is simply the product of their individual PDFs:

$$
p_{X, Y}(x, y)=\frac{1}{2 \pi \sigma^{2}} e^{-\left(x^{2}+y^{2}\right) / 2 \sigma^{2}}
$$

Using the definition of a PDF, we obtain:

$$
P(x \leq X \leq x+d x, y \leq Y \leq y+d y)=\frac{1}{2 \pi \sigma^{2}} e^{-\left(x^{2}+y^{2}\right) / 2 \sigma^{2}} d x d y
$$

The equation $Z=\sqrt{X^{2}+Y^{2}}$ can represent a conversion from Cartesian coordinates $X$ and $Y$ to the radius $Z$ in polar coordinates. In such a case, the area $d x d y$ in Cartesian coordinates equals the area $z d z d \theta$ in polar coordinates. Therefore, by transformation of probability spaces, we obtain:

$$
P(z \leq Z \leq z+d z, \theta \leq \Theta \leq \theta+d \theta)=P(x \leq X \leq x+d x, y \leq Y \leq y+d y)
$$




$$
\begin{gathered}
\Rightarrow P(z \leq Z \leq z+d z, \theta \leq \Theta \leq \theta+d \theta)=\frac{1}{2 \pi \sigma^{2}} e^{-\left(x^{2}+y^{2}\right) / 2 \sigma^{2}} z d z d \theta \\
\Rightarrow p_{Z, \Theta}(z, \theta)=\left(\frac{z}{\sigma^{2}} e^{-\left(z^{2}\right) / 2 \sigma^{2}}\right)\left(\frac{1}{2 \pi}\right)
\end{gathered}
$$

Since the joint PDF of $Z$ and $\Theta$ can be separated into two functions that are dependent only on the corresponding random variables; $Z$ and $\Theta$ are independent. Therefore, the PDF of $\mathrm{Z}$ is:

$$
p_{Z}(z)=\frac{z}{\sigma^{2}} e^{-\left(z^{2}\right) / 2 \sigma^{2}}, z \geq 0
$$

which is the well known formulation of the Rayleigh PDF. 


\section{Chapter 3}

\section{Beampattern Effects}

For an ideal radiation source that is omnidirectional, the location of the scatterer will have no effect on the system's echo amplitude. However, the systems used in many real world applications may have highly directional beams, requiring the need to account for directionality, as summarized below.

\subsection{General Theory}

If the scatterer is assumed to be randomly located in the beam, the directionality of the beampattern can have a significant effect on the echo amplitude distribution [7]. As seen in (2.2), the net echo amplitude for the system is the product of system constants, the amplitude of the beampattern, and the scattering amplitude. In this thesis, the effects of the beampattern are taken into account by assuming a random distribution for the location of the scatterer. Assuming an axisymmetric aperture for

$b$ (one variable), the change of variables formula for functions of random variables can be used to find the probability distribution of the beampattern $b$. For a function 
that is not monotonic, [3] this formula is:

$$
p_{b}(b)=\sum_{l=1}^{n(b)} \frac{p_{\theta}(\theta)}{\left|\frac{d b_{l}}{d \theta}\right|_{\theta}}
$$

where the summand is summed over each $l^{\text {th }}$ piecewise monotonic section $b_{l}$ of the beampattern and $n(b)$ is the number of sections, all of which are contiguous. For the case of $k a=2 \pi$, the beampattern has a single sidelobe in addition to the mainlobe. This corresponds to three piecewise monotonic sections (Fig. 3-1).

This expression was used by Ehrenberg and in the below analysis for a rotationally symmetrical beampattern. However, it also applies for the case of an asymmetrical beampattern (such as for a rectangular aperture) when the location of the scatterer is limited to a single plane (and, hence, requires only a single variable, $\theta$, to describe the location).

The symmetric nature of the beampattern allows an analysis of the probability distribution of $\theta$ between $0 \leq \theta \leq \pi / 2$. The prolate spheroid is assumed to be found at any location in the MRA plane with equal probability. Therefore, the probability distribution is constant over the range and can be described as:

$$
p_{\theta}(\theta)=\frac{2}{\pi}, 0 \leq \theta \leq \pi / 2, \quad \text { MRA plane }
$$

If the location of the center of the prolate spheroid is not assumed to be restricted to the MRA plane, then the prolate spheroid may be found anywhere in $2 \pi$ half-space with equal probability. Assuming that the prolate spheroid will always be found at a fixed distance from the aperture, we obtain:

$$
P(\theta \leq \Theta \leq \theta+d \theta, \psi \leq \Psi \leq \psi+d \psi)=\sin (\theta) d \psi d \theta
$$

where the angles $\theta$ and $\psi$ can be visualized as the polar and azimuthal components 
of a spherical coordinate system, respectively.

To find the probability distribution of $\theta$, we can now integrate over all possible values of $\psi$ to obtain:

$$
p_{\theta}(\theta)=\sin (\theta), 0 \leq \theta \leq \pi / 2, \quad \text { half }- \text { space }
$$

In the following sections, this thesis will focus on the case where the center of the prolate spheroid is restricted to the MRA plane and the probability distribution of $\theta$ is constant (3.2).

When measuring the echo amplitude of an object, the amplitude of the beampattern is a composite of beampattern effects on the incident and scattered waves. That is,

$$
b=b_{i} b_{r}
$$

The case of far field radiation in a time-invariant medium is considered, therefore, the property of reciprocity is assumed to hold. A related assumption is the linearity of the medium, where the parameters of the medium are invariant under changes in the values of fields or waves. It should be noted that reciprocity theorems generally do not hold for the case of nonlinear media, where the frequency $\omega$ of the wave is not conserved. Reciprocity implies equality of power transfer from the transmitter to the receiver, if the roles of the receive and transmit elements are reversed [16]. In this thesis, the assumption of a monostatic system implies that:

$$
b_{i}^{2}=b_{r}^{2}=b
$$




\subsection{Applications to Circular Aperture}

For a circular aperture, the shape of the beampattern varies according to the parameter $k a$ where $k$ is the acoustic wavenumber and $a$ is the radius of the transducer. Thus, the net amplitude of the two-way beampattern at an azimuthal coordinate $\theta$ is [17]:

$$
b(\theta)=\left(\frac{2 J_{1}(k a \sin (\theta))}{2 \pi \sin (\theta)}\right)^{2}
$$

where $J_{1}$ is the Bessel function of the first kind of order 1 .

Equations (3.2) and (3.7) are subsituted into (3.1) to derive the beampattern PDF. This formula is evaluated numerically in Matlab to obtain a plot for the PDF on a log-log scale (Fig. 3-2). This curve may be interpreted as the PDF of the magnitude of the scattering amplitude from a smooth sphere or point scatterer in the beam. Note the singularity at the maximum echo amplitude. This calculation involved the scatterer lying in the MRA plane and $p_{\theta}(\theta)$ being a constant. For a scatterer randomly located throughout the beampattern, $p_{\theta}(\theta)=\sin (\theta)$ and that section of the echo amplitude PDF is a line with constant slope and no singularity.

The results of these calculations for a circular aperture are presented in Figs. 3-1 - 3-4 below. Fig. 3-1 plots the result of the two-way beampattern amplitude from (3.7) for the case of $k a=2 \pi$ with $\theta$ ranging between 0 and $\pi / 2$. There are three piecewise monotonic sections that are summed in (3.1), indicated by $l=1,2$ and 3. In addition, there are three points where the differential of the beampattern $b$ goes to zero, indicated by A, B and C. According to (3.1), these points will lead to singularities in the plot of the beampattern PDF, which is shown in Fig. 3-2. The beampattern PDF is shown here under the assumption that the scatterer is confined to the MRA plane, i.e. the PDF of the angle $\theta$ is a constant.

In Fig. 3-3, the scatterer is assumed to lie at any point within the $2 \pi$ half-space. In this case, the random variable $\theta$ is distributed as the sine function. The highest 
amplitude of the beampattern is found at the center of the mainlobe, i.e. $\theta=0$. The differential of the beampattern amplitude at this peak is zero, leading to the singularity labeled ' $\mathrm{C}$ ' in Fig. 3-2. However, in Fig. 3-3, the PDF of $\theta$ goes to zero in the limit as the value of $\theta$ goes to zero. Since the PDF and the differential of the beampattern amplitude decay at the same rate, $p_{b}$ as calculated from (3.1) tends towards a constant instead of infinity. Therefore, the singularity 'C' is absent in Fig. 3-3, whereas the sidelobe singularities corresponding to ' $\mathrm{A}$ ' and ' $\mathrm{B}$ ' are still present. In all of the figures discussed above, it is assumed that $k a=2 \pi$.

In Fig. 3-4, the beampattern PDFs for different values of $k a$ are shown. These PDFs are qualitatively similar to the $k a=2 \pi$ case, with an increased number of singularities that correspond to the higher number of sidelobes present in these beampatterns. However, the singularity corresponding to point ' $A$ ' in Fig. 3-1 does not appear in the graphs. Although such a singularity is present in theory, it does not appear in the curve calculated in Matlab. This is because of the limited resolution of a discrete data set in Matlab, as well as the narrow range of amplitudes over which the singularity appears. The main lobe for $k a \geq 6 \pi$ is significantly narrower when compared to the $k a=2 \pi$ case. As a result, the differential of the beampattern with respect to angle approaches zero very rapidly, and this is not reflected in the PDF calculation. 


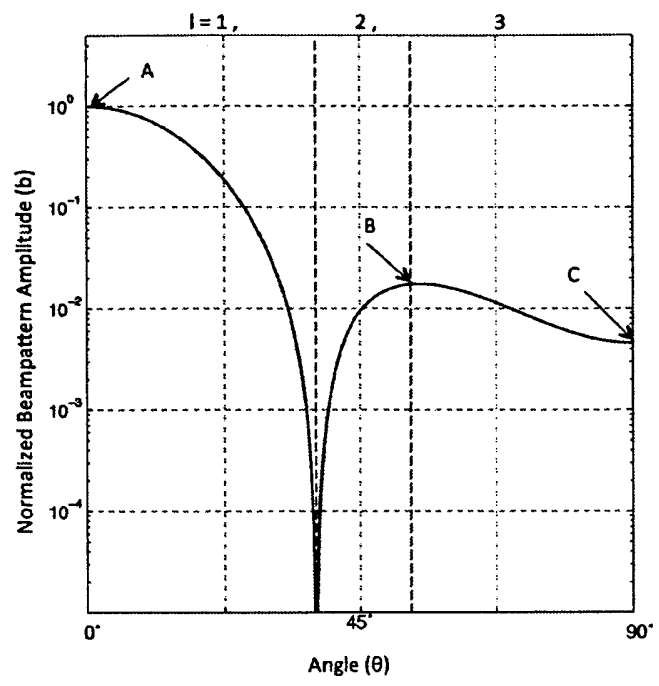

Figure 3-1: The amplitude of a two-way composite beampattern (3.7) for a circular aperture with $k a=2 \pi$. This corresponds to a beamwidth of approximately $30^{\circ}$. The points of interest marked $\mathrm{A}, \mathrm{B}$ and $\mathrm{C}$ indicate regions where the slope of the curve is zero. These points correspond to the singularities labeled in Fig. 3-2. The sections of the plot labeled 1,2 and 3 correspond to piecewise monotonic sections $b_{l}$ of the beampattern amplitude.

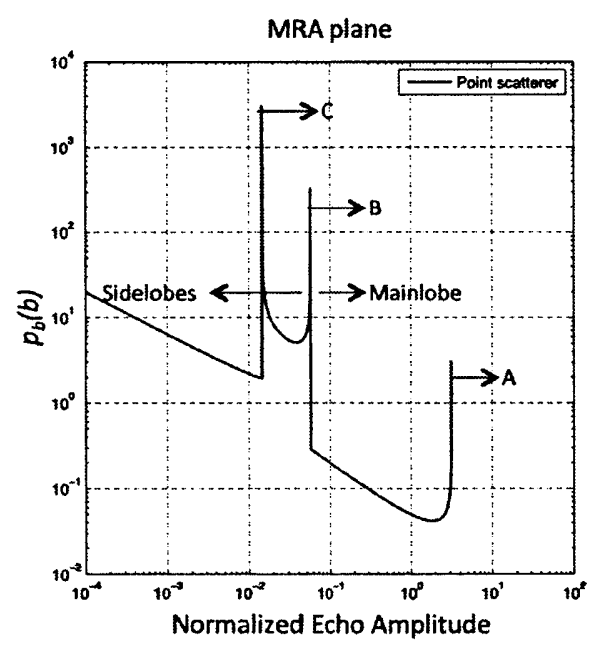

Figure 3-2: The PDF of the two-way composite beampattern amplitude for a circular aperture with $k a=2 \pi$. The scatterer is assumed to be limited to the MRA plane, i.e. $p_{\theta}=$ constant. The PDF for narrower beams is qualitatively similar, as shown in Fig. 3-3. The singularities marked A, B and C correspond to points of zero slope in the beampattern amplitude curve (Fig. 3-1). 


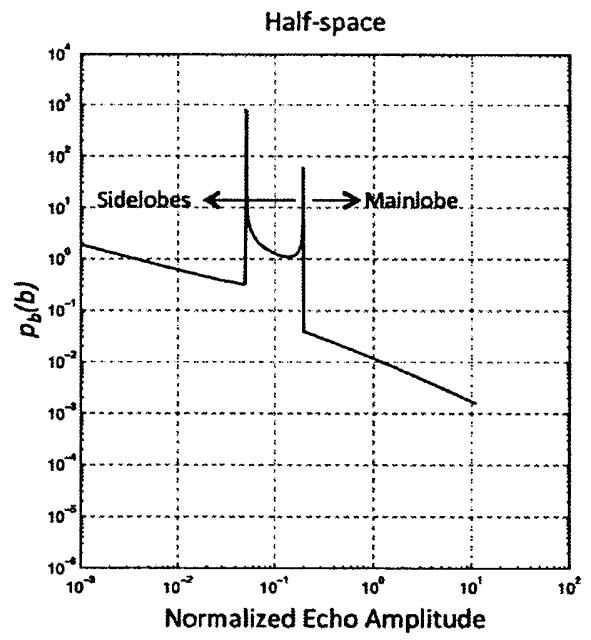

Figure 3-3: The two-way composite beampattern for a circular aperture with ka $=$ $2 \pi$. In contrast to Fig. $3-2$, the scatterer is assumed to lie in the half-space, i.e. $p_{\theta}=\sin (\theta)$. Note the absence of a singularity corresponding to ' $\mathrm{A}$ ' in Fig. 3-2. 

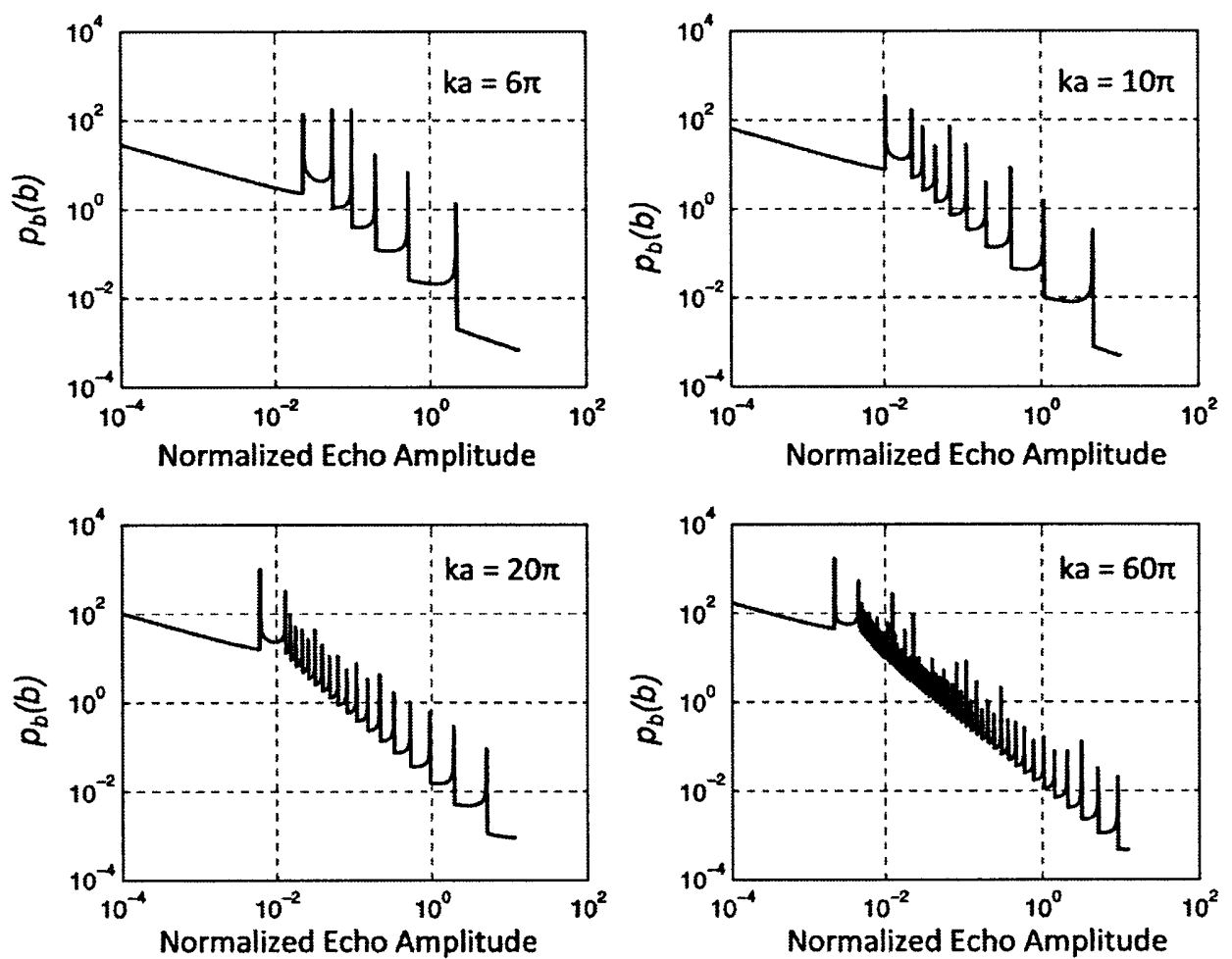

Figure 3-4: The two-way composite beampatterns for circular apertures with various values of $\mathrm{ka}$, demonstrating the increase in the number of singularities due to additional sidelobes. The center of the prolate spheroid is assumed to be confined to the MRA plane. 


\section{Chapter 4}

\section{Scattering by Prolate Spheroids}

\section{(No Beampattern Effects)}

The problem of finding the scattering amplitude of a scatterer is well studied in the literature [6]. The specific case of a prolate spheroid scatterer has been analyzed for the cases of electromagnetic [18] and acoustic [19] incident waves.

The prolate spheroid shape has broad applicability in practical applications of remote sensing, both acoustic and electromagnetic. As one example, scattering from swimbladders of fish has been shown to be an important component of the acoustic broadband backscattering amplitude from fish. This organ can be reasonably simulated using a prolate spheroid scatterer as a model [20].

In many applications, the orientation of the prolate spheroid may not remain constant with respect to the incident waves. In the example discussed above, the fish being insonified may change the direction in which they are swimming between successive pings from the sonar system. This change in orientation will significantly affect the backscattering amplitude, and increase variability in amplitude between successive pings. This variation can be modeled by assuming a random distribution for the angle of orientation $\gamma$ of the prolate spheroid with respect to the incident 
waves (Fig. 1-1).

In this chapter, the scattering amplitude of a smooth prolate spheroid for the deterministic case of fixed orientation is first given. In the subsequent section, the stochastic effects of random orientation are analyzed by deriving the probability distribution of the scattering amplitude, given that the PDF of $\gamma$ is known prior to the calculation. Finally, the case of a prolate spheroid with surface roughness is analyzed. The stochastic effects of the roughness are calculated heuristically and the PDF of a rough, randomly oriented prolate spheroid is presented.

\subsection{Deterministic Scattering (Fixed Orientation, Smooth Spheroid)}

As discussed earlier in Chapter 2, the scattered pressure wave $P_{s}$ can be described in terms of the incident pressure $P_{i}$, wavenumber $k$, and range $r(2.1)$. The interaction between the scatterer and the incident wave is assumed to be in the geometric regime. That is, the wavelength of the incident wave is much smaller than the radius of curvature of the prolate spheroid at the point of incidence. This allows the use of the Kirchhoff approximation for the deterministic scattering amplitude [21]:

$$
f_{s s}=\frac{i}{\lambda} \iint_{A} \hat{k}_{i n c} \cdot \hat{n} e^{2 i \vec{k} . \overrightarrow{A_{A}}} d A
$$

where $\lambda$ is the wavelength of the incident wave, $\hat{k}_{i n c}$ is the unit vector in the direction of the incident wave, $\hat{n}$ is the outward normal to the surface at the point of incidence, and $r_{A}$ is the distance between the source and the surface.

The use of the Kirchhoff approximation requires two important assumptions [21]. Firstly, the field and derivatives across the surface are exactly the same as they would be in the absence of the object. Secondly, the field and its derivative are identically 
zero in the geometric shadow of the object under consideration. These assumptions will be approximately true only if the size of the object being considered is much larger than the wavelength of the incident wave. Therefore, the analysis presented in this thesis is limited to the case of the geometric scattering regime, where both assumptions hold true.

Using the above approximations leads to an analytical, frequency-independent formulation for the scattering cross-section of a prolate spheroid. The magnitude of the scattering amplitude of the prolate spheroid is the square root of the scattering cross-section and is given by [20]:

$$
\left|f_{s s}\right|=\frac{c}{2} \frac{\sin ^{2}\left(\tan ^{-1}\left(b_{1} / c t a n \gamma\right)\right)}{\cos ^{2} \gamma}
$$

Where $\gamma$ is the angle between the incident beam and the plane that is normal to the polar axis of the prolate spheroid (Fig. 1-1). When $\gamma=0^{\circ}$, the spheroid is broadside-on with respect to the incident wave and $\gamma=90^{\circ}$ represents the end-on case. $c$ is the length of the semi-major axis of the prolate spheroid and it has semiminor axes of equal length, $b_{1}=b_{2}$. The length and width of the scatterer are thus $2 c$ and $2 b_{1}=2 b_{2}$, respectively. The aspect ratio of the prolate spheroid is defined to be $c / b_{1}$ (or, equivalently, length/width).

The target strength can be derived from the scattering amplitude using:

$$
T S=10 \log \left|f_{s s}\right|^{2}
$$

For the case of broadside incidence [20], (4.2) reduces to:

$$
\left|f_{s s}\right|_{0}=\frac{c}{2}
$$

The results of (4.2) are shown in Fig. 4-1 below. The scattering amplitude for 
values of $\gamma$ between 0 and $\pi / 2$ is shown for prolate spheroids with aspect ratios ranging from 1:1 (sphere) to 10:1 (elongated). The spheroids are assumed to enclose a constant volume with varying aspect ratio, therefore, the peak broadside scattering amplitude of the 10:1 prolate spheroid is greater than that of the sphere. The sphere itself is symmetric over all axes, resulting in a constant scattering amplitude with respect to $\gamma$. The scattering is shown to be more directional with increasing aspect ratio.

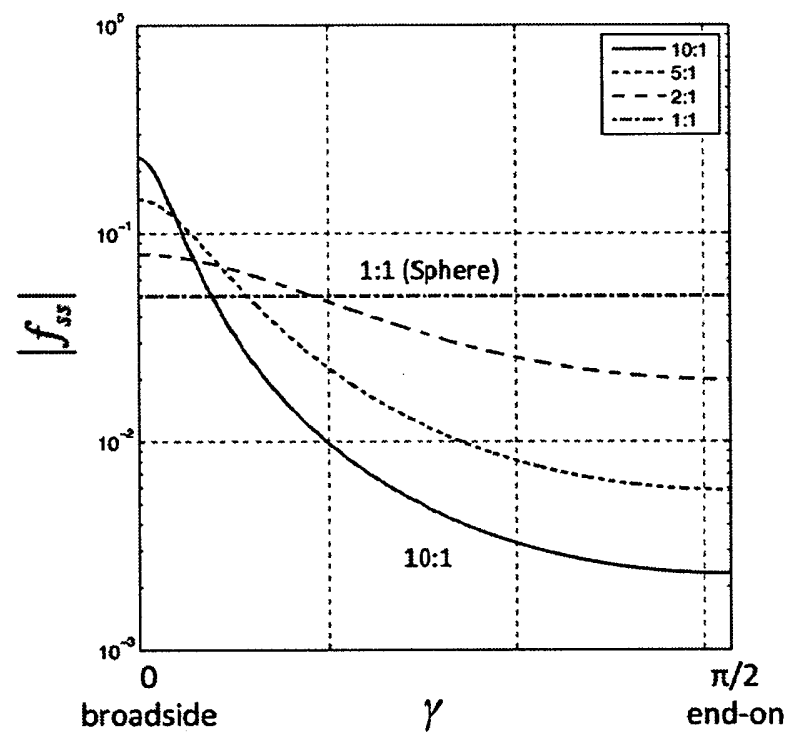

Figure 4-1: Scattering amplitude with varying angle of incidence for prolate spheroids of constant volume and different aspect ratios.

\subsection{Stochastic Effects (Random Orientation, Smooth Spheroid)}

The prolate spheroid may be at any orientation $\phi$ with equal probability. The axis of rotation of the prolate spheroid is fixed and perpendicular to the MRA plane of the transducer. Therefore, $\phi$ is a uniform random variable between 0 and $\pi / 2$, which is independent of $\theta$, the location of the prolate spheroid in the beam. Including 
the range of values between $\pi / 2$ and $2 \pi$ is unnecessary due to the symmetry of the scatterer's shape. Thus, the PDF of $\phi$ is:

$$
p_{\phi}(\phi)=\frac{2}{\pi} \quad \text { for } 0 \leq \phi \leq \pi / 2
$$

However, the scattering amplitude of the prolate spheroid is dependent on the angle relative to the transducer, $\gamma$. This angle is dependent on both the location and orientation of the prolate spheroid. Using elementary geometry:

$$
\gamma=\theta+\phi
$$

The symmetry of the prolate spheroid allows a direct substitution of the distribution of $\gamma$ for that of $\phi$ in (4.5), since the PDF is being calculated between 0 and $2 \pi$ in both cases. The scattering amplitude functions as dependent on $\gamma$ and $\phi$ will be identical except for a shift along the $\mathrm{X}$-axis that is modulo $2 \pi$. Since there is no change in the shape of the amplitude function, the PDF of the scattering amplitude derived from $\gamma$ will be identical to one derived from $\phi$. Therefore, the probability distribution of $f_{s s}$ can be derived in a manner similar to the beampattern PDF in (3.1):

$$
p_{s s}\left(f_{s s}\right)=\frac{p_{\gamma}(\gamma)}{\left|\frac{d f_{s s}}{d \gamma}\right|_{\gamma}}
$$

The above formula has a simpler form than (3.1) since the function $f_{s s}$ is monotonic.

The results of substituting (4.2) in (4.7) are illustrated in Fig. 4-2 below. The PDF of the scattering amplitude of a sphere is not shown. Since the scattering amplitude of a sphere is constant with respect to $\gamma$ (Fig. 4-1), the PDF of the scattering amplitude would be a delta function at that constant value. As the aspect ratios of the prolate spheroids increase and their volume is kept constant, the difference between 
the maximum (broadside) and minimum (end-on) amplitudes increases. This results in a PDF that spans a progressively larger range of amplitudes with increasing aspect ratio.

The differential of the scattering amplitude goes to zero near $\gamma=0$ (broadside) and $\pi / 2$ (end-on). Since this differential is present in the denominator of the PDF expression (4.7), and the numerator is a constant value, singularities are present at the maximum and minimum amplitudes of each scattering amplitude PDF.

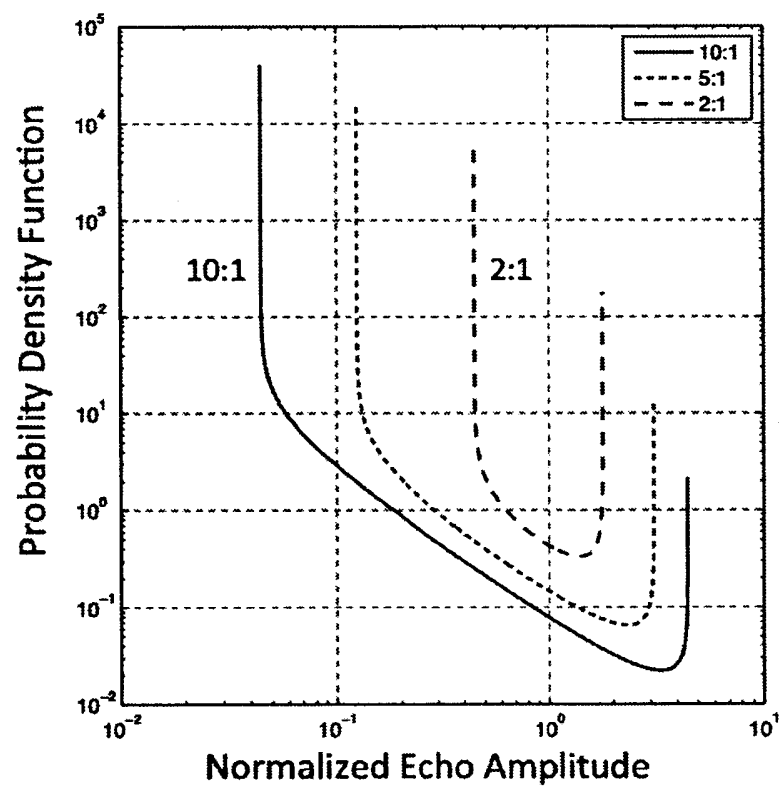

Figure 4-2: PDFs of scattering amplitude for smooth prolate spheroids that are randomly and uniformly oriented in a plane that also contains the incident field. Beampattern effects are not included.

\subsection{Stochastic Effects (Random Orientation, Rough Spheroid)}

It has been shown that scattering at short wavelengths by randomly rough bounded objects at fixed orientations can be Rayleigh distributed $[9,10]$. One of the earliest 
studies on this subject was written by Eckart in 1953, involving scattering of sound by the sea surface [22]. Due to the time-varying, random changes in the sea surface, developing mathematical models with analytical formulas for surface scattering was difficult. Using a statistical approach, Eckart obtained approximate analytical formulas for the sea surface scattering coefficients of short- and long-wavelength acoustic waves. Using the Kirchhoff approximation for the short wavelength case and assuming that the height of the sea surface is a Gaussian random variable, Eckart obtained the following result for the mean scattered field due to an incident plane wave:

$$
\langle R\rangle=e^{-2 k^{2} h^{2} \cos ^{2} \theta}
$$

where $\mathrm{R}$ is the reflection coefficient of the sea surface, $\mathrm{h}$ is the standard deviation of variations in sea height and $\theta$ is the angle of incidence of the acoustic signal.

After further manipulation and approximations, Eckart was able to derive an expression for the scattering per unit area of the sea surface, assuming that surface waves followed a bivariate Gaussian distribution:

$$
\sigma=\frac{1}{8 \pi \alpha \beta} e^{-1 / 2\left[(a / \alpha c)^{2}+(b / \beta c)^{2}\right]}
$$

where $\sigma$ is the scattering coefficient, $-\alpha^{2}$ and $-\beta^{2}$ represent the zero-lag autocorrelations of the Gaussian distributions representing variations in sea height along the Xand Y-axes, respectively. The coefficients $a$ and $b$ represent the sums of the components of the incident and scattered vectors in the $\mathrm{X}$ - and $\mathrm{Y}$-directions, and they are related to $k h$.

For the case of uniform roughness around the object, the echo from the smooth spheroid at a fixed orientation is assumed to be modulated with a Rayleigh distributed random variable $[9,10]$. This is expected to be further modulated by an equation related to (4.8) and (4.9) with an exponential dependence upon $k^{2} h^{2}$. However, the 
PDF is normalized with respect to the root mean square (rms) amplitude. As a result of the normalization, the exponential modulation drops out and has no effect on the PDF.

Using the formula for the distribution of the product of two random variables (derived in Section 2.1.2), the echo from the smooth prolate spheroid modulated by a Rayleigh distributed random variable is:

$$
p_{r s}\left(\left|f_{r s}\right|\right)=\int_{\left|f_{r s}\right|}^{\infty} \frac{1}{x} p_{r a y}(x) p_{s s}\left(\frac{\left|f_{r s}\right|}{x}\right) d x
$$

Where $p_{r s}$ and $p_{s s}$ are the echo amplitude distributions of rough and smooth prolate spheroids, respectively, and $p_{\text {ray }}$ is the standard Rayleigh PDF. Due to the complex analytical description of $p_{s s}$ and the presence of $1 / x$ as a function argument within the integral, finding an analytical solution for (4.10) is very difficult.

As in the case of the beampattern PDF, a solution is numerically calculated in Matlab and the result is shown in Fig. 4-3. This figure plots the PDF of rough prolate spheroids of varying aspect ratios with respect to normalized amplitude. As illustrated in the plot, an increase in the aspect ratio of the prolate spheroid moves the tail of the PDF away from that of the Rayleigh distribution, thus increasing the degree to which the echo PDF is non-Rayleigh.

Unlike the echo PDFs of the smooth spheroids shown in Fig. 4-2, the PDFs of the rough spheroids lack singularities. This is due to the continuous and unbounded nature of the Rayleigh PDF. Modulating the scattering amplitude of smooth prolate spheroid with a Rayleigh distributed random variable has the effect of "smoothing over" the singularities, resulting in continuous, smooth PDFs.

Fig. 4-3 includes the special case corresponding to a rough sphere, or a rough prolate spheroid with an aspect ratio of unity. In this case, the scattering amplitude of the smooth sphere is constant and independent of the orientation $\gamma$ of the 
sphere. Therefore, the PDF of the scattering amplitude from the smooth sphere is a delta function, normalized to unit amplitude. The resulting PDF of the scattering amplitude of the rough sphere $p_{r s}$ is identical to the Rayleigh distribution, since multiplication by a constant has no effect on a normalized probability distribution.

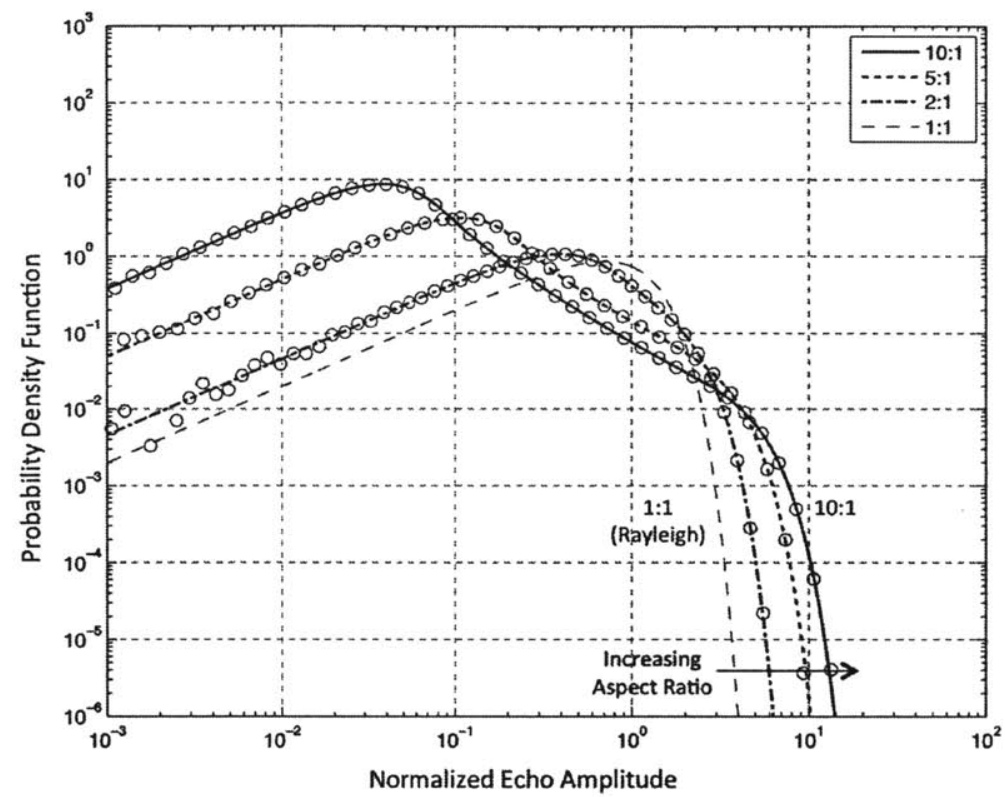

Figure 4-3: PDFs of scattering amplitude for randomly oriented rough prolate spheroids of varying aspect ratios, without beampattern effects. The circular markers represent the results of Monte Carlo simulations for validation, discussed later in the thesis. 


\section{Chapter 5}

\section{Scattering by Prolate Spheroids with Beampattern Effects}

The probability distributions of the beampattern and the echo amplitude of a randomly oriented rough prolate spheroid have been determined in chapters 3 and 4 , respectively. As shown in chapter 2 , the total echo amplitude of the system is the product of the beampattern and the scattering amplitude (without beampattern effects) of the rough prolate spheroid. The effects of the system constants are not considered, as the PDFs are normalized with respect to their rms values. The normalization results in PDFs that are invariant under changes to the system constants.

The PDF of the overall echo amplitude for the system of a cylindrical aperture and a randomly oriented and located prolate spheroid can now be derived using (4.10), (3.1), and (3.7). The result of the calculation is presented in Figs. 5-1 - 5-3. For comparison, the cases of a smooth spheroid, a Rayleigh scatterer and a point object are also included, with beampattern effects applied to each scatterer. Finally, a Rayleigh distribution is shown to illustrate the degree to which the above distributions are non-Rayleigh.

From Fig. 5-1, some important characteristics of the echo amplitude PDFs are ap- 


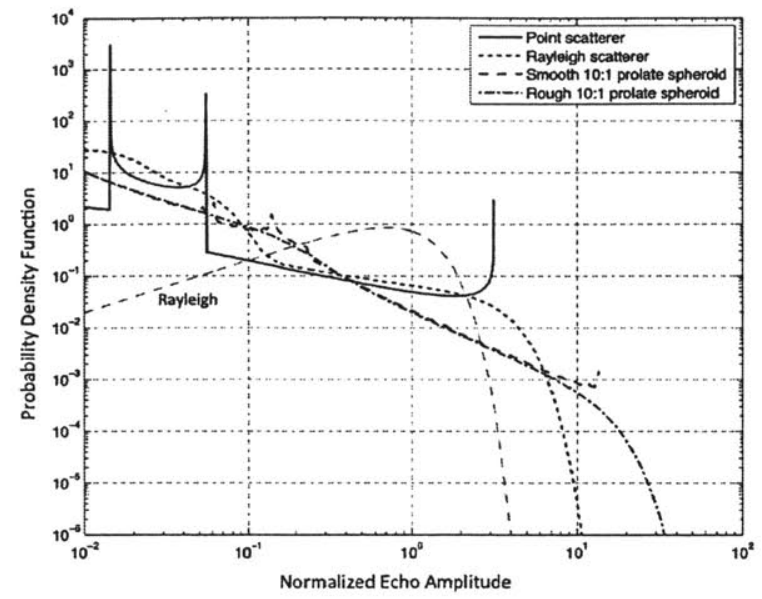

Figure 5-1: Scattering amplitude PDFs for various scatterers, with beampattern effects.

parent. The point scatterer and smooth prolate spheroid PDFs have a finite maximum value and the probability of obtaining a stronger echo are zero. This is intuitively correct, as the point scatterer returns the strongest possible echo when $\theta=0^{\circ}$ and the smooth prolate spheroid does so when both $\phi$ and $\theta$ are $0^{\circ}$. For the case of a Rayleigh scatterer, the beampattern's echo amplitude is modulated by a Rayleigh random variable with no upper bound. Therefore, there is a small non-zero probability of obtaining an echo amplitude above any positive number. Similarly, the rough prolate spheroid modulates the finite echo amplitude of a smooth prolate spheroid, with a Rayleigh random variable. The resulting PDF is once again positive up to infinity.

The PDFs of the echo amplitudes of rough prolate spheroids that are randomly oriented, taking into account beampattern effects, are shown in Fig. 5-2. As is the case when beampattern effects are not taken into account (Fig. 4-3), prolate spheroids with higher aspect ratios have PDFs that are increasingly non-Rayleigh in nature. In particular, the tails of the PDFs deviate further from the tail of the Rayleigh PDF with increasing aspect ratio. 
For a rough prolate spheroid of a given aspect ratio, taking beampattern effects into account increases the degree to which the amplitude PDF is non-Rayleigh (Figs. 4-3 and 5-2). This is especially true at lower values of the echo amplitude. When beampattern effects are not included, the PDF increases steadily until it reaches a mode. After this point, the curve decreases monotonically, with a tail that is dependent on the aspect ratio of the prolate spheroid. When beampattern effects are taken into account, the complexity of the PDF function increases significantly. The presence of a mode in the distribution is no longer obvious, and PDFs of different aspect ratios intersect at up to five points. Without beampattern effects, the PDFs of spheroids of two different aspect ratios always intersect at two points.

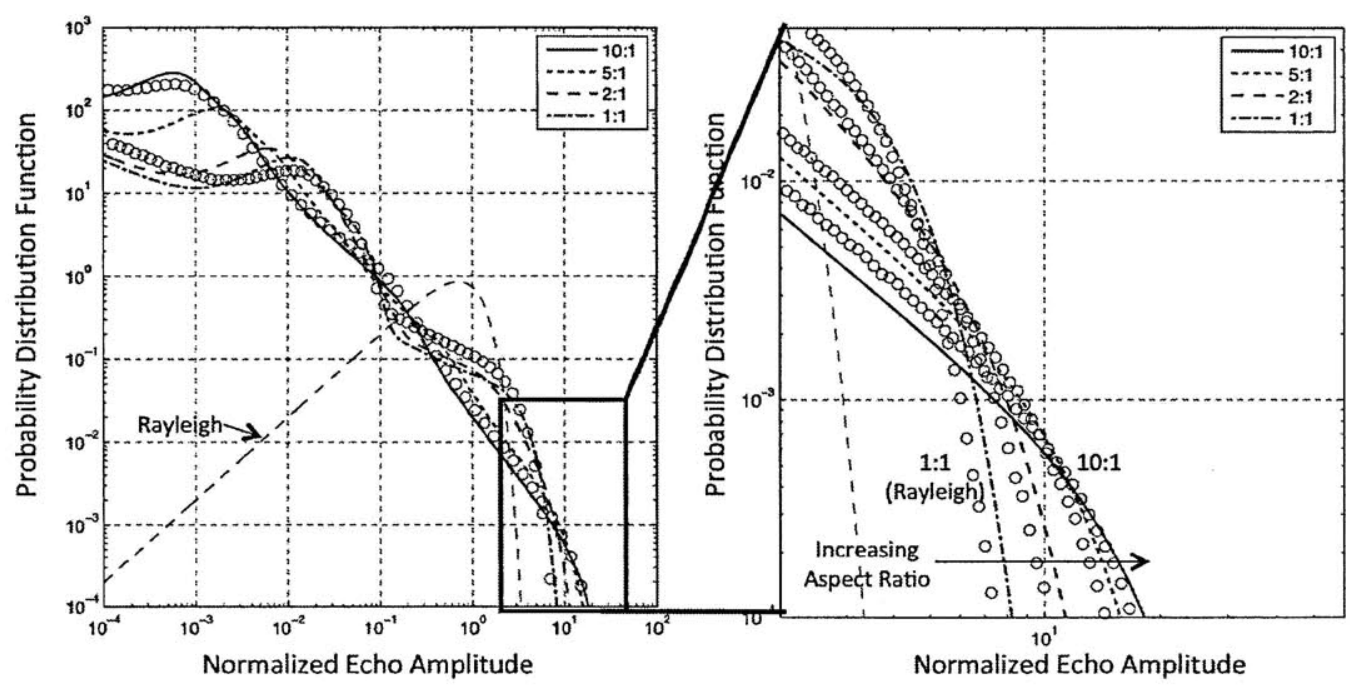

Figure 5-2: Echo amplitude distributions for rough prolate spheroids of varying aspect ratios, with beampattern effects. The data points are the results of Monte Carlo simulations for validation, discussed later in the paper. The plot on the right provides a zoomed-in view focusing on the tails of the PDFs for prolate spheroids of various aspect ratios.

For practical applications, inspecting the probabilities of false alarm (PFA) for each of the cases discussed previously may be insightful. In particular, it is apparent from Fig. 5-3 that the tails of the PFAs for each case are non-Rayleigh to different extents. The rough prolate spheroid with a 10:1 aspect ratio has the most strongly 
non-Rayleigh echo PFA. Compared to a smooth prolate spheroid, the difference in probabilities is large above a normalized echo amplitude of 20 . The smooth prolate spheroid is, in turn, more strongly non-Rayleigh than the Rayleigh scatterer with beampattern effects.

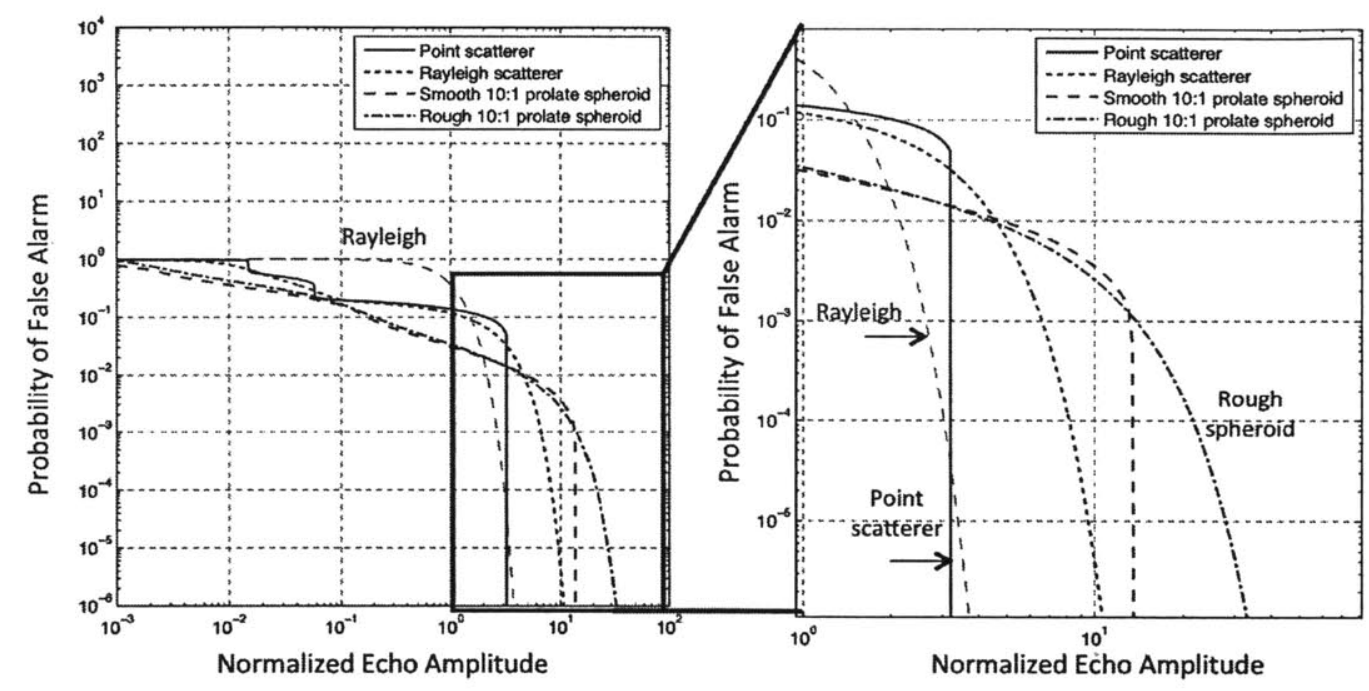

Figure 5-3: Plot of the scattering amplitude PFAs for various types of scatterers (corresponding to Fig. 5-1), as well as a plot focused on the tails of these PFAs. 


\section{Chapter 6}

\section{Monte Carlo Validation and Computational Issues}

Due to the difficulty of obtaining analytical solutions to the integrals described in chapters $2-5$, these integrals are solved numerically in the MatLab programming environment. Since MatLab operates on discrete data sets with finite resolution, it is important to validate the results to ensure that accuracy is not compromised. The validation procedure is performed using Monte Carlo methods described in Section 6.1 below.

Modeling continuous functions with discrete data sets also lead to various computational issues, which are described and addressed in Section 6.2. The foremost problem encountered during validation was caused by the singularities in the beampattern PDFs. The presence of these singularities necessitated adaptive sampling methods to obtain high accuracy and successfully verify the results of the numerical integration. 


\subsection{Monte Carlo Validation}

To verify the probabilistic calculations, the rough prolate spheroid case was simulated using Monte Carlo methods. Each of the variables associated with the characteristics of the prolate spheroid is generated according to the appropriate random distribution. The orientation of the prolate spheroid $\phi$ and its location in the beampattern $\theta$ are both uniform between 0 and $\pi / 2$. An inbuilt MatLab routine was used to generate these random variables. The problem of constructing sets of pseudorandom numbers from deterministic information is well studied, and MatLab's results fulfill certain statistical tests, such as negligible correlation between random numbers in the same set. Therefore, MatLab's inbuilt rand(...) function is used without any modifica-

tions. The echo amplitude of the prolate spheroid is then calculated according to the generated values of $\phi$ and $\theta$. To account for the roughness, the echo amplitude is multiplied by a Rayleigh random variable. MatLab also has an inbuilt routine for generating random numbers that are distributed according to the Rayleigh PDF. This routine uses the inverse sampling method to derive Rayleigh distributed random numbers from a uniform distribution, and it was used without modifications in this research.

In most practical situations, the tail of the probability of false alarm will be of the most interest. To obtain an accurate simulation of the tail of the curve, many events with probabilities below $10^{-6}$ must be evaluated. To ensure a statistically significant number of samples even at very low probabilities, several million prolate spheroid coordinates are generated. The data points obtained for their echo amplitudes are then binned with logarithmic bin widths. The number of spheroids assigned to each bin is normalized by the width of the bin and the whole curve is normalized to ensure the area below the probability distribution is 1 . This result is then compared to the numerically evaluated analytical formulas as shown in Figs. 4-3 and 5-2. As evident in these plots, there is close agreement between the Monte Carlo and 
analytical methods. The beampattern PDF for a cylindrical transducer contains a number of singularities that occur when the differential of the beampattern function with respect to $\theta$ approaches zero. For a PDF derived from Monte Carlo simulations, it is difficult to obtain an accurate estimate of the integral of the function over a singularity. This explains why the match between analytical and Monte Carlo data in Fig. 5-2 is not perfect.

\subsection{Computational Issues}

All of the above computations are conducted using discrete data sets in Matlab. The discrete nature of the data means that additional care must be taken while performing operations meant for continuous functions, especially derivatives and integrals. In particular, the integral in the formulation for the product of two random variables is difficult to evaluate with high precision (2.12). Ideally, Matlab's inbuilt routine for performing Simpson's method may be used to provide a very good approximation for an integral. However, this routine requires a function that can be evaluated at arbitrary values. Due to the process of normalization and the lack of a simple analytical formulation for the functions being used, the distribution can only be computed over a finite set of amplitudes. The values required for evaluating the product integral must be interpolated from this set.

As shown in Section 2.1.2, the product of two random variables is a commutative operation. In the context of (4.10), this means that the order of the prolate spheroid echo PDF and the Rayleigh 'roughness' PDF can be interchanged. Since the function with $\frac{f_{r s}}{x}$ as a parameter will require interpolation, we choose this function to be the Rayleigh PDF. This is because of the well known analytical formulation of a Rayleigh PDF, which Matlab's inbuilt routines can evaluate with very high precision.

While validating the beampattern PDFs using Monte Carlo methods, the pres- 
ence of singularities required the use of adaptive sampling. The singularities in the theoretical beampattern distribution arise at the maximum amplitude of the sidelobe, where the slope changes sign. The theoretical PDF has a very sharp peak near this amplitude that Monte Carlo simulation with fixed bin widths cannot account for. The area under the singularity leads to a significant difference in the theoretical and simulated curves once they are normalized. This issue was corrected by sharply reducing bin widths in the vicinity of known singularities in the beampattern PDF. The bin widths were reduced until the area under the singularity converged to a constant value. This correction resulted in well matched theoretical and simulated curves.

The comparison of the analytical and Monte Carlo methods for calculating the beampattern PDF is illustrated in Fig. 6-1 for the case of $k a=2 \pi$. Here, the 'analytical' method refers to numerical evaluation of the analytical formula (3.1). As seen in the plot, the singularities that are apparent in the analytical plot (solid line, (3.1)) are not accurately reflected in the Monte Carlo simulation (circles, (3.7)). Therefore, the area contained in a narrow region around the singularity is much larger for the analytical curve, when compared to the Monte Carlo curve. Since the total area under the PDF is normalized to unity, the Monte Carlo curve lies above the analytical curve for all values other than at the singularities.

The result of the Monte Carlo simulation when using the adaptive sampling technique described above is shown in Fig. 6-2. In this case, the width of the bins is initially constant on a logarithmic scale at all points on the curve. The algorithm, implemented in Matlab, analyzes the analytically generated PDF curve to find singularities. The bins that lie near the singularity are then subdivided up to fifty times in order to allow the Monte Carlo simulation to capture the singularity with better accuracy. As seen in Fig. 6-2, the peaks in the Monte Carlo curve are almost as high as those in the analytically generated PDF. As a result, there is very little difference overall between the two methods, and the analytical procedure is successfully 
validated.

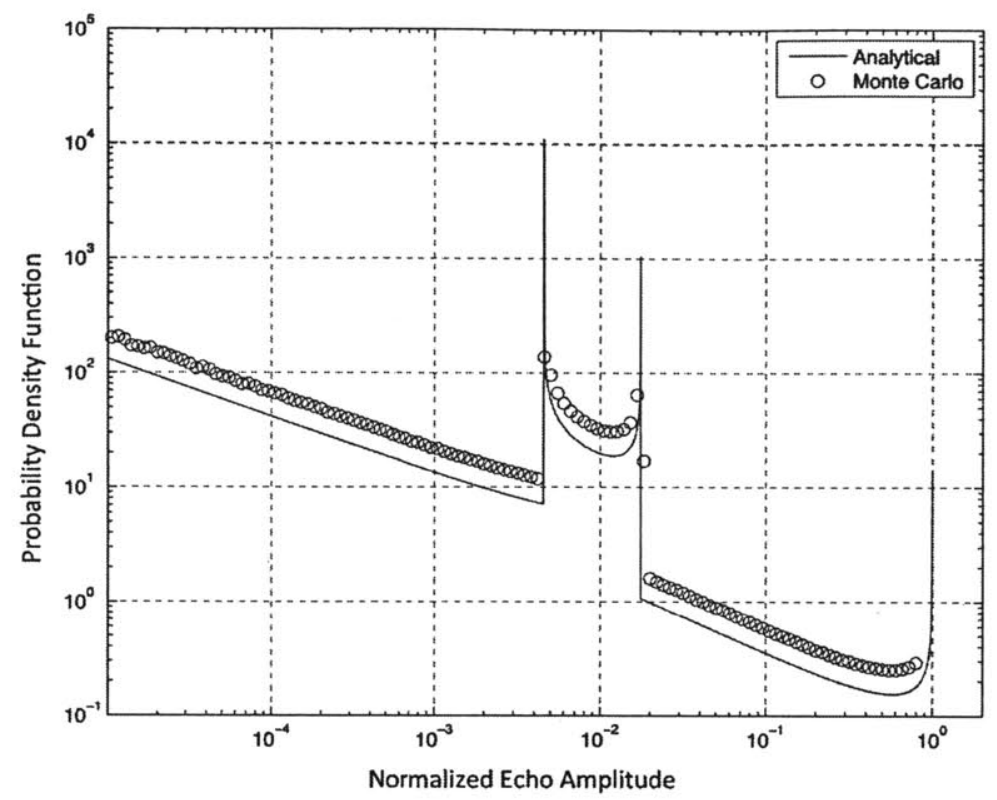

Figure 6-1: Beampattern PDF associated with two-way composite beampattern for a circular aperture with $\mathrm{ka}=2 \pi$. The numerically calculated curve (solid line) based on analytical formulas (3.1) and (3.7), is compared to a Monte Carlo simulation of a scatterer with constant scattering amplitude in the beam (circles). The simulation data is divided into bins with equal width on a log scale. 


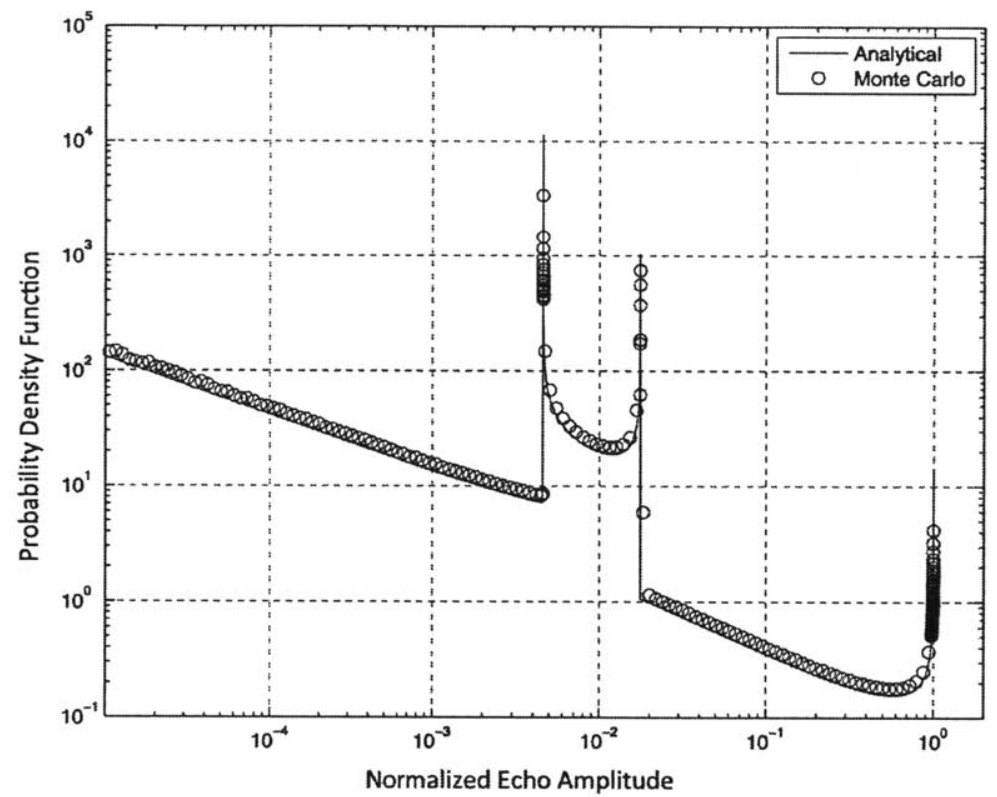

Figure 6-2: Beampattern PDF associated with two-way composite beampattern for a circular aperture with $\mathrm{ka}=2 \pi$, using (3.1) and (3.7). The numerically calculated curve (solid line) is compared to a Monte Carlo simulation of a scatterer with constant scattering amplitude in the beam (circles). The simulation data is adaptively divided into bins with varying width. The width of bins is much lower near the singularities present in the curve. 


\section{Chapter 7}

\section{Summary and Conclusion}

The primary contributions of this thesis are related to the approach of determining echo statistics from first principles rather than inferring these statistics from previously obtained experimental data. In contrast to previous studies that used generic statistical functions to describe the echo statistics and whose parameters were based principally or solely on data, the formulation described herein rigorously relates key physical characteristics of the scatterer and parameters of the transceiver system to the shape of the echo PDF. The formulation takes into account previously published studies on the beampattern effects of the sensing system, which are randomized due to the random location of the scatterer. Thus, this physics-based approach is predictive and can be applied to a wide range of types of scatterers and transceivers. A summary of the main contributions is presented below.

- The echo statistics for a randomly oriented prolate spheroid are derived, first without beampattern effects.

- Smooth prolate spheroid, varying aspect ratios

- Rough prolate spheroid, varying aspect ratios

- The echo statistics of the overall system are now derived, taking into account 
beampattern effects.

- Smooth prolate spheroid with beampattern effects, varying aspect ratios

- Rough prolate spheroid with beampattern effects, varying aspect ratios

- The theoretical predictions were validated through Monte Carlo simulations.

In addition, the following calculations were made:

- A comparison between beampattern PDFs when the scatterer is restricted to the MRA plane versus when it is assumed to lie at any location in the $2 \pi$ half plane.

- A comparison between beampattern PDFs for different values of $k a$.

- PDFs of various types of scatterers, with beampattern effects are explored. In particular, differences in the slopes of the tails of each curve and the degree to which they are non-Rayleigh are demonstrated. These properties are shown to be dependent on the presence of surface roughness and the aspect ratio of the prolate spheroid.

- The PFAs of the scatterers analyzed above are also shown, with qualitatively similar results.

The predictions show that the echo PDF from the spheroid in the beam is strongly non-Rayleigh. The degree to which it is non-Rayleigh and, specifically the tail of the distribution, increases with increasing complexity of the scatterer (point scatterer through rough spheroid) as well as increasing aspect ratio of the spheroid. It had long been known that beampattern effects and number of scatterers contribute to the non-Rayleigh nature of echoes. This study shows that the complexity of the scatterer also contributes to the non-Rayleigh nature of the echo and must be taken into account. 
Also, the calculations were limited to the direct path geometry (that is, no waveguide effects) and the case in which the orientation and location of the spheroid were varied uniformly over all angles in the same plane that also contains the MRA. The two important cases of a waveguide environment and arbitrary range of orientation and location are outside the scope of this current study. However, it has been shown in the first case that the effects of a long-range multi-path environment in which there are both reflections directly from the scatterer and from the boundaries, the echo will tend toward Rayleigh for many paths [23]. Also, it is anticipated in the second case that once the orientation of the prolate spheroid span all angles and the scatterer is located anywhere in the beam (i.e., neither quantity is limited to being in a plane), the results would be qualitatively similar. The results could differ significantly when the orientations and/or locations are limited to arbitrary ranges of angles. 


\section{Appendix A}

\section{Matlab Code}

This section includes selected Matlab code files written by the author that were critical to the research described in this thesis. The source code was run using Matlab R2011b.

\section{A.1 ellipsoid.m}

This function calculates the scattering amplitude and the PDF of the scattering amplitude of a prolate spheroid with given eccentricity $e_{a c}$ over one million uniformly spaced values of $\theta$ between 0 and $\pi / 2$. The prolate spheroid is assumed to have a minor axis of length $0.1 \mathrm{~m}$. However, the final dimensions of the spheroid are not relevant as the final result is normalized. The function implicitly assumes that the scattering amplitude is non-negative and monotonic over all calculated values of $\theta$. The PDF of $\theta$ is assumed to be uniform over the range, and the PDF of the scattering amplitude is derived using (4.7).

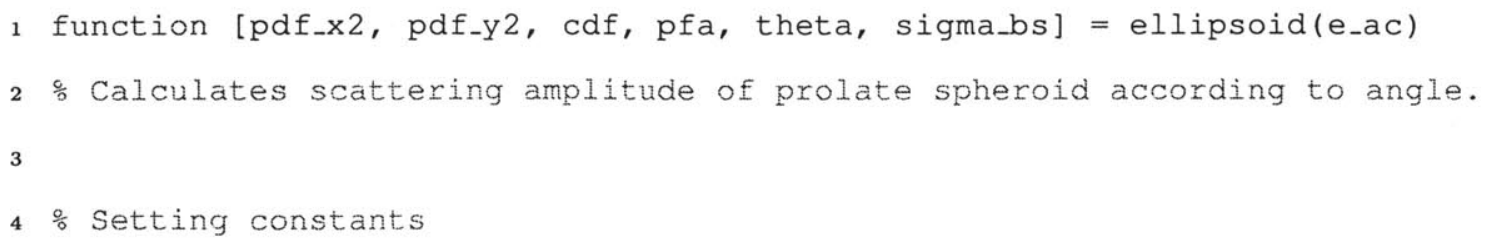




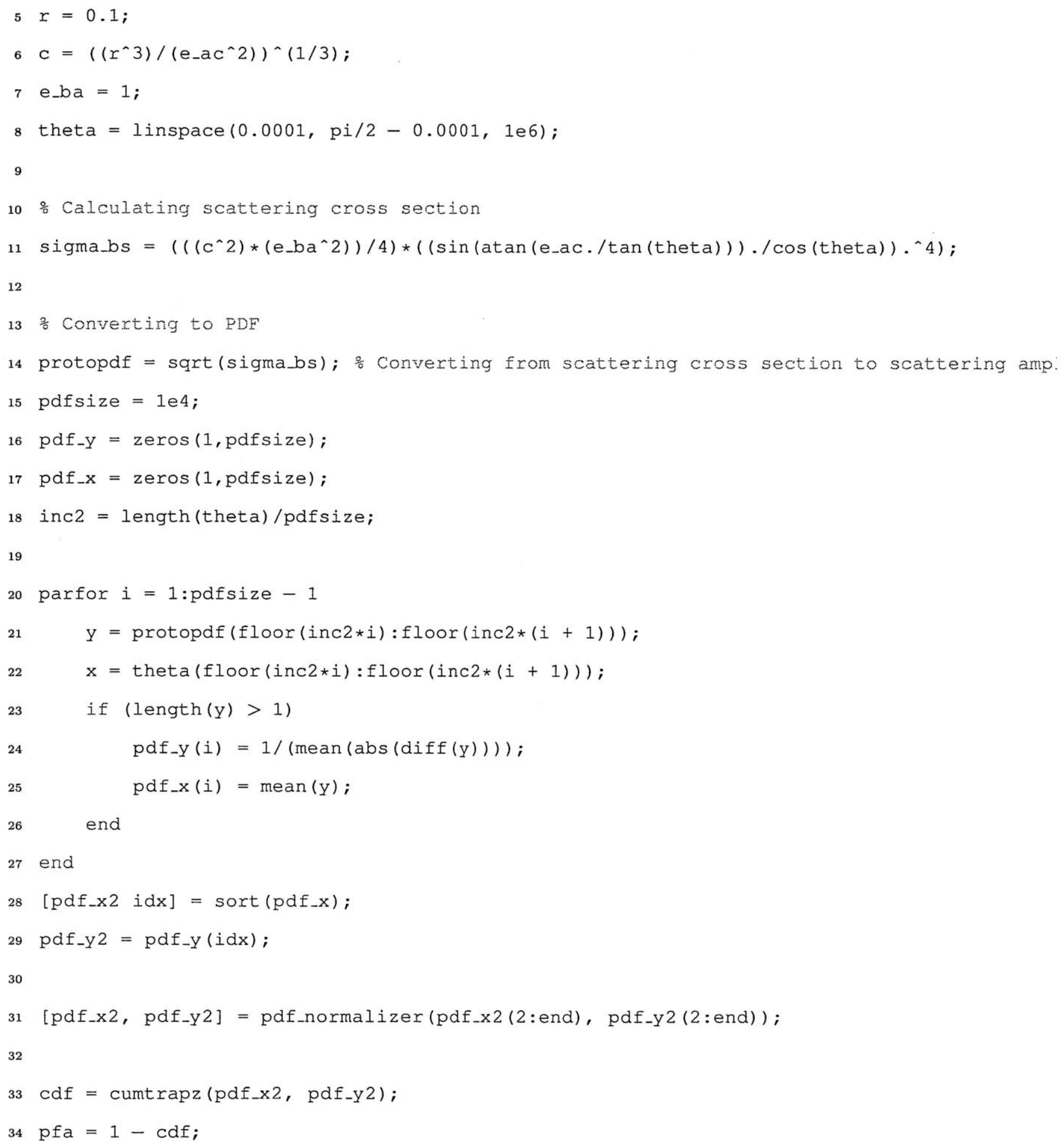




\section{A.2 cyl_bp_num2.m}

This function calculates the PDF of the beampattern amplitude generated by a cylindrical piston transducer of radius $a$ that is emitting sound with wavenumber $k$. The function accepts the product of both values as the sole input parameter. Due to symmetry, the beampattern is calculated for values of $\theta$ between 0 and $\pi / 2$. The PDF of the beampattern is derived using (4.7).

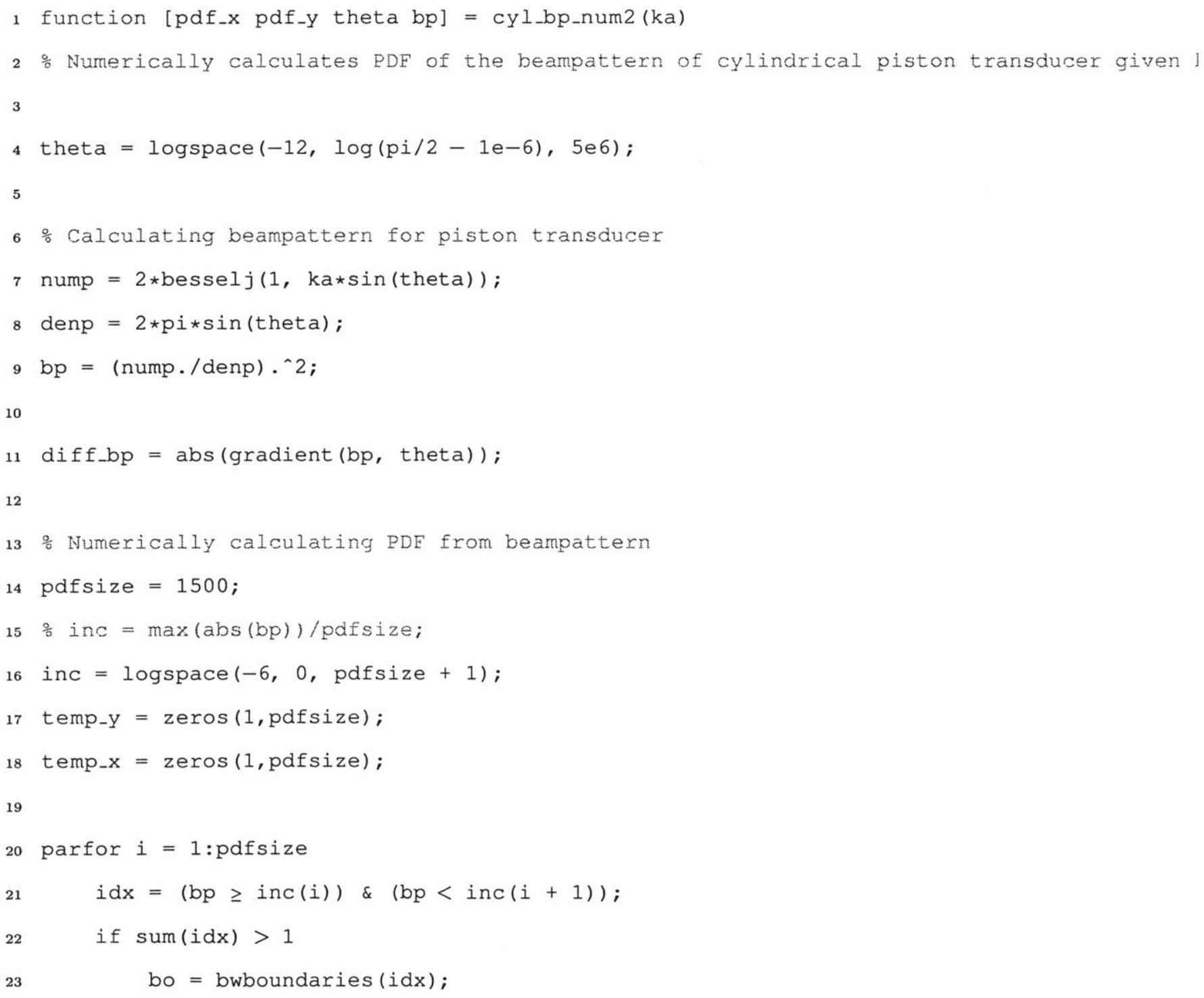




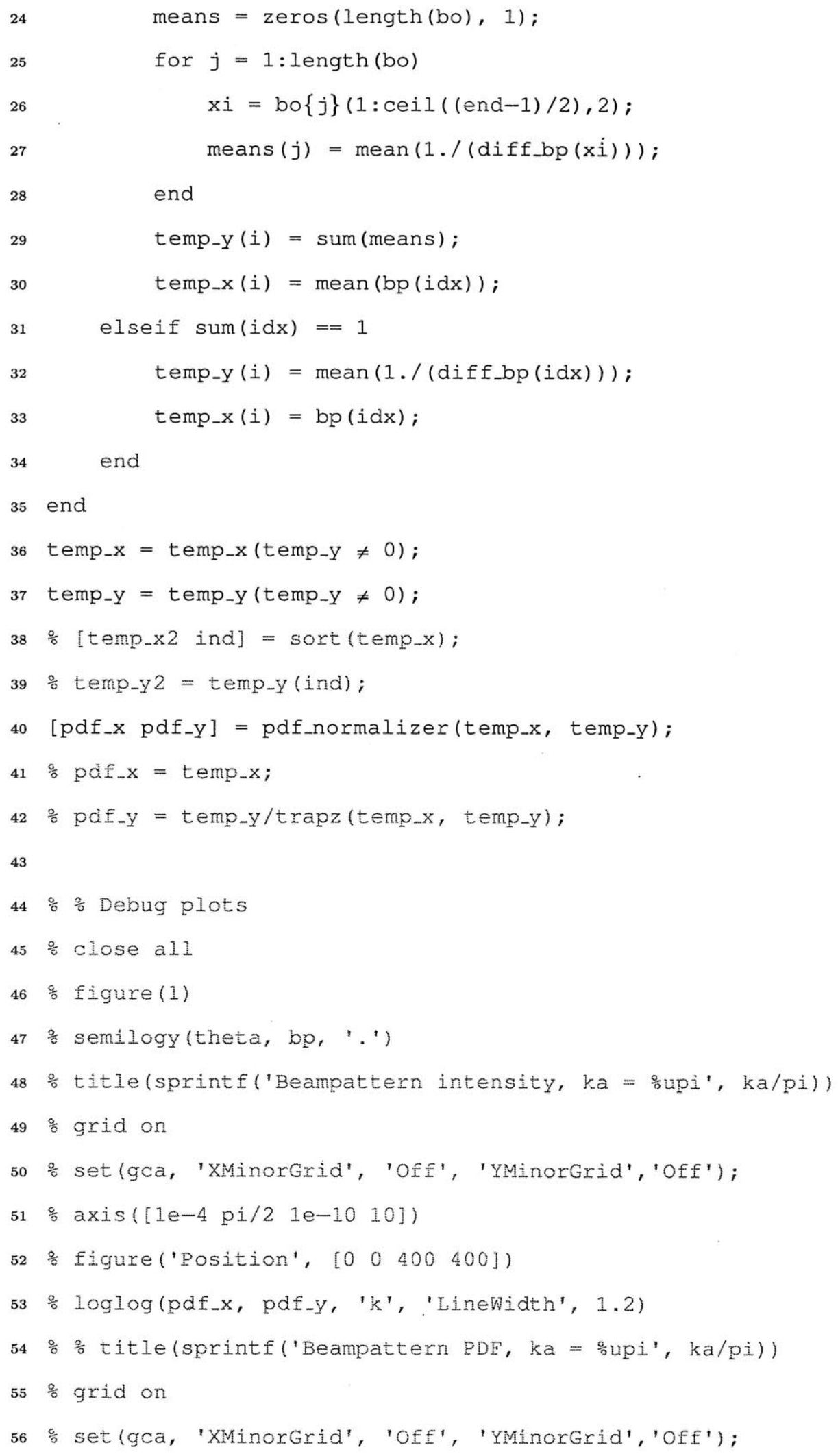




\section{A.3 prosph_simulation.m}

This function implements a Monte Carlo simulation in order to generate the PDF of the echo amplitude of a system with a rough prolate spheroid scatterer of given eccentricity and a transducer with fixed $k a=2 \pi$, using (3.7) and (4.2). A total of five million "spheroids" are simulated and the echo amplitude of each object is sorted into bins that have uniform width on a logarithmic scale. This binned data is then utilized to verify the outcome of the analytical calculations performed in this thesis.

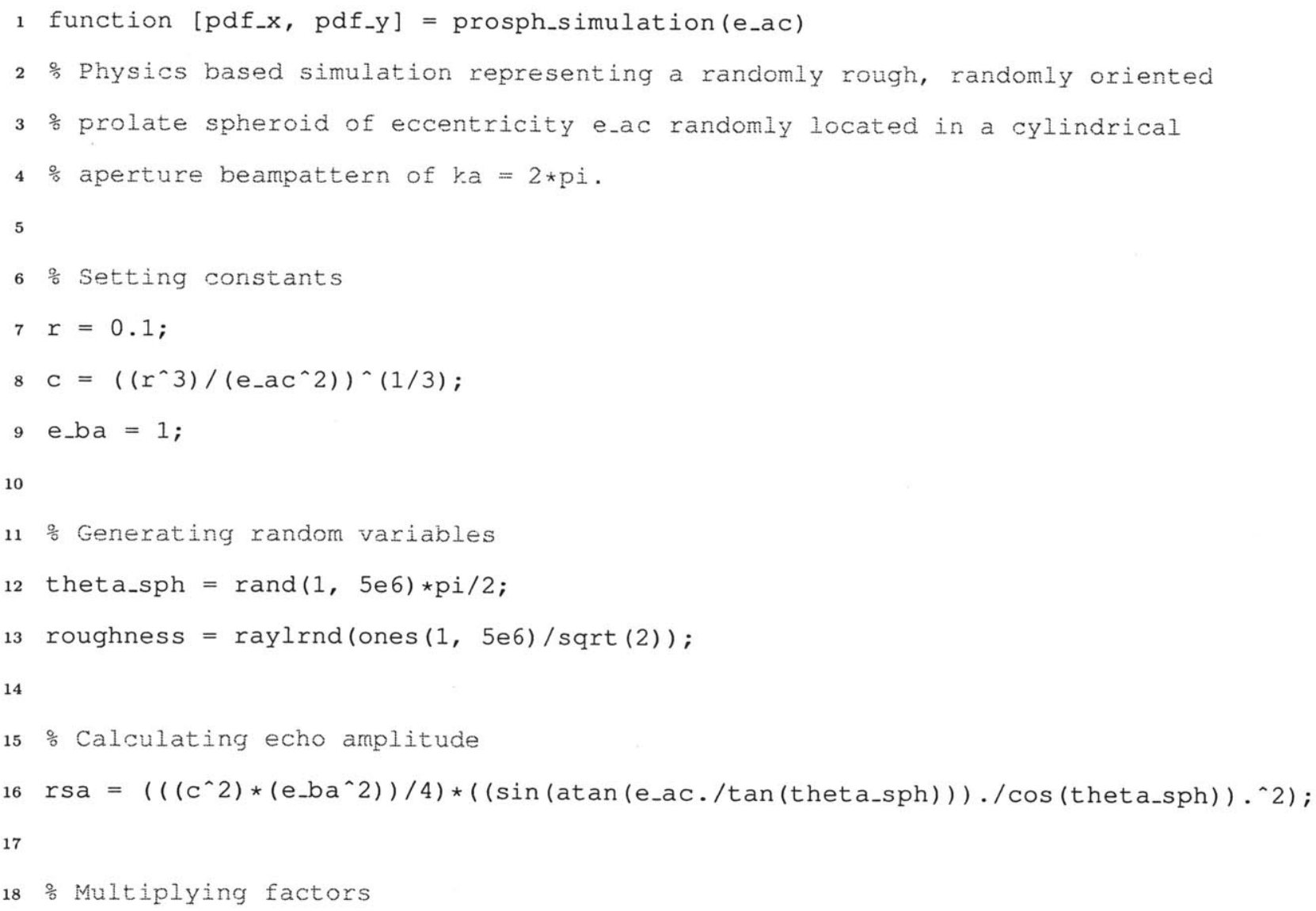


\% Binning data

[pdf_x, pdf_y] = logbinner (data, 300, 0);

\section{A.4 pdf_multiplier2.m}

This function finds the PDF of the product of two random variables with known PDFs using (2.13). The second PDF that is entered as a parameter to this function is interpolated in order to perform discrete numerical integration with a rational expression in the function argument. The primary loop is parallelized for computational efficiency. This function should be called with Matlab's inbuilt matlabpool routine for faster computation on multicore systems.

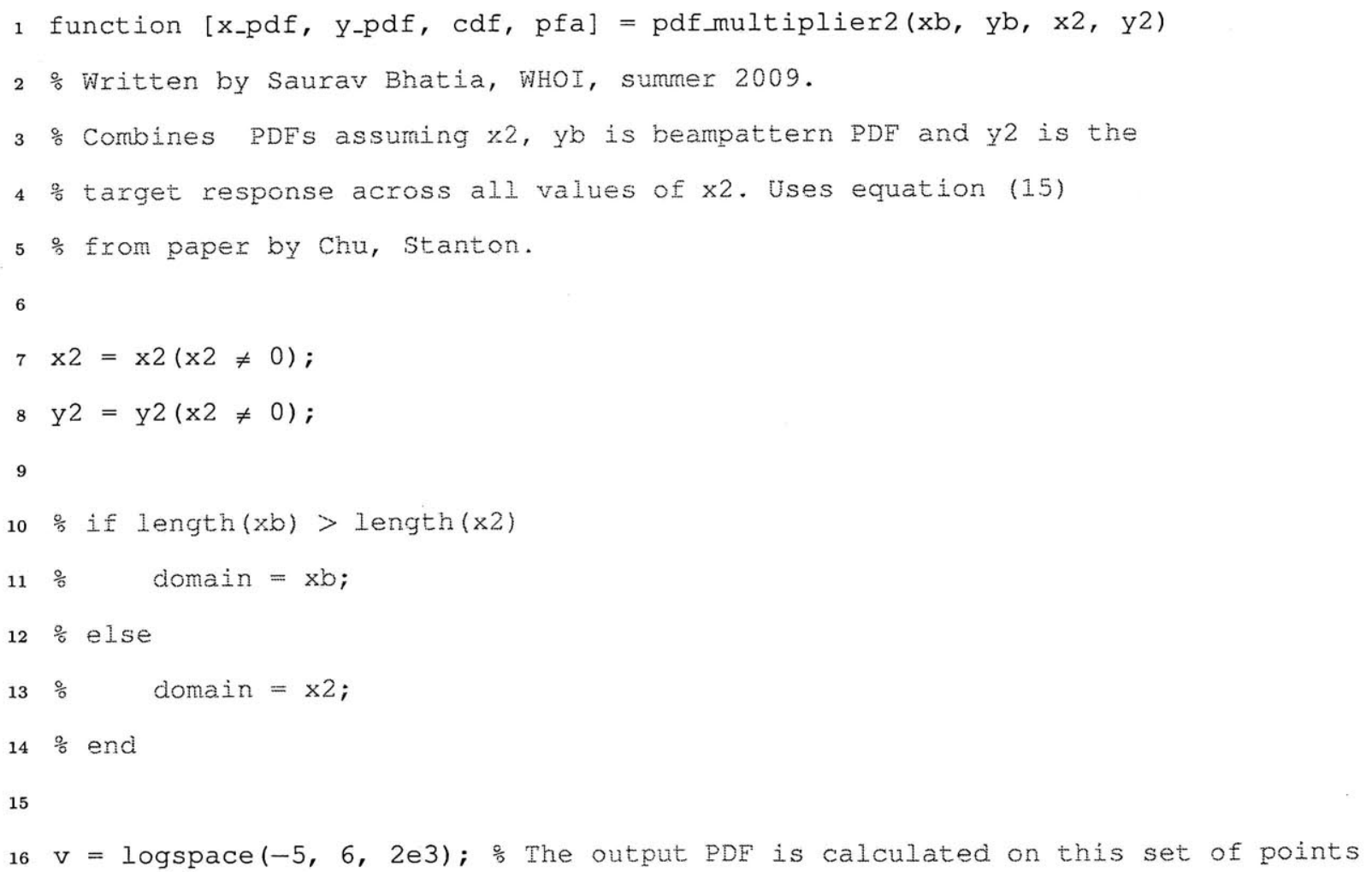




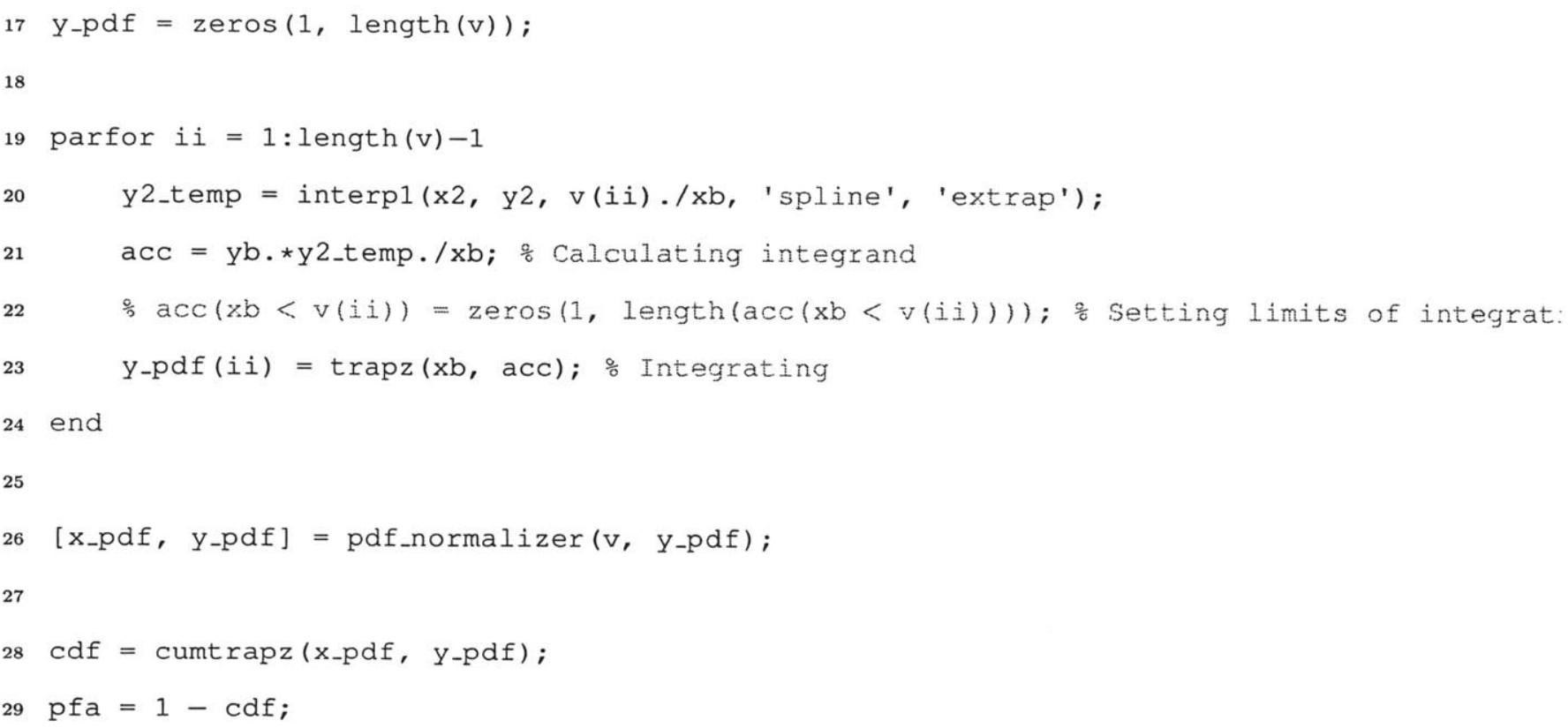

\section{A.5 roughprolatespheroid.m}

This function provides examples of calls to the other functions listed in this appendix and generates Figs. 5-2 and 5-3.

1 close all

2 Load('data/tsdata.mat')

3 load('data/pdf_prolatespheroid.mat')

4 matlabpool open 8

5

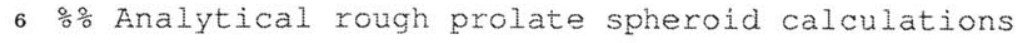

$7\left[x_{-} 10_{-} 1 r, y_{-10} 1 r\right.$, cdf10r, pfal0r $]$ = roughellipsoid $(0.1)$;

8 [x_5_1r, y_5_1r, cdf5r, pfa5r] = roughellipsoid(0.2);

9 [x_3_1r, y_3_1r, cdf3r, pfa3r] = roughellipsoid(1/3);

$10\left[x_{-}-1 r, y-2-1 r, \operatorname{cdf} 2 r, \operatorname{pfa} 2 r\right]=$ roughellipsoid(0.5);

11

$12 \%$ Numerical rough prolate spheroid calculations 


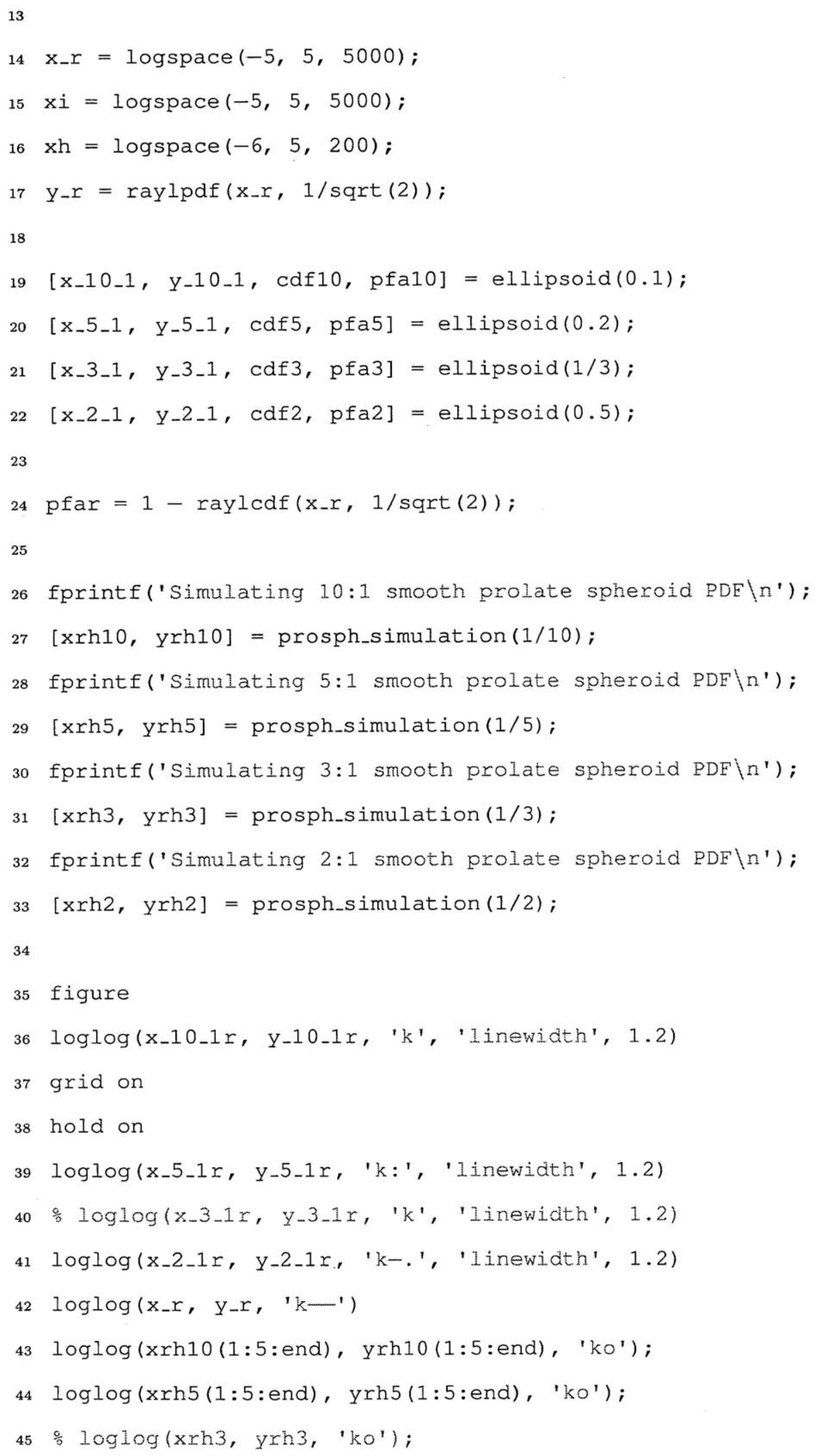




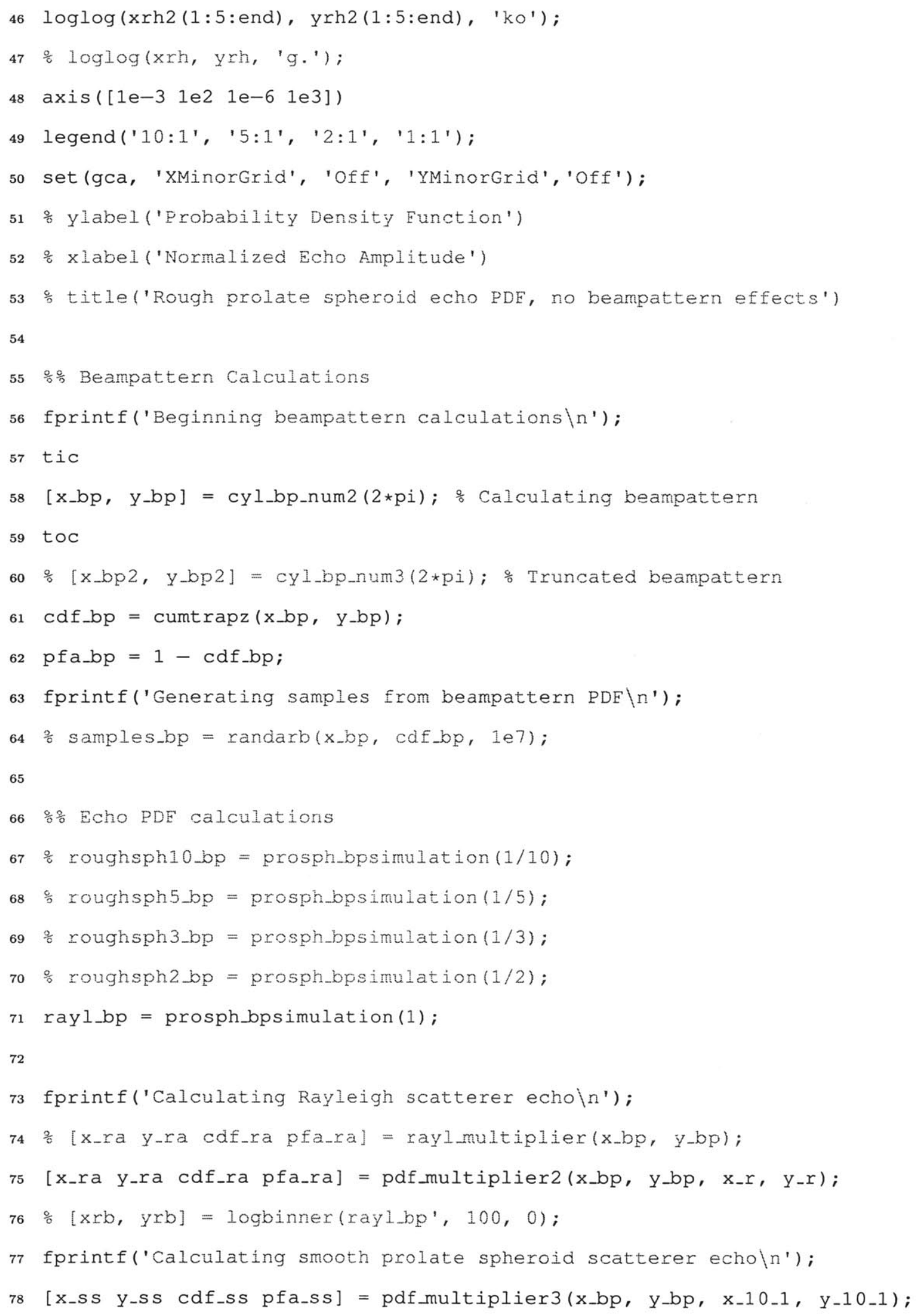


94

95

96

97

98

99

fprintf('Calculating 10:1 rough prolate spheroid scatterer echoln');

$[\mathrm{xrb10}, \mathrm{y} r \mathrm{~b} 10]=$ prosph_bpsimulation $(1 / 10)$;

[x_rs10 y_rs10 cdf_rs10 pfa_rs10] = pdf_multiplier2(x_bp, y_bp, x_10_1r, y_10_1r);

fprintf('Calculating 5:1 rough prolate spheroid scatterer echoln');

$[\mathrm{xrb5}, \mathrm{yrb5}]=$ prosph_bpsimulation $(1 / 5)$;

[x_rs5 y_rs5 cdf_rs5 pfa_rs5] = pdf_multiplier2 (x_bp, y_bp, x_5_1r, y_5_1r);

fprintf('Calculating 3:1 rough prolate spheroid scatterer echoln');

$[\mathrm{xrb3}, \mathrm{yrb3}]=$ prosph_bpsimulation $(1 / 3)$;

[x_rs3 y_rs3 cdf_rs3 pfa_rs3] = pdf_multiplier2(x_bp, y_bp, x_3_1r, y_3_1r);

fprintf('Calculating 2:1 rough prolate spheroid scatterer echoln');

$[\mathrm{xrb2}, \mathrm{yrb2}]=$ prosph_bpsimulation $(1 / 2) ;$

[x_rs2 y_rs2 cdf_rs2 pfa_rs2] = pdf_multiplier2(x_bp, y_bp, x_2_1r, y_2_1r);

$[\mathrm{xrb}, \mathrm{yrb}]=$ prosph_bpsimulation(1);

음 Plotting results

figure ('Position', [1 400400 400])

$\log \log \left(x \_r s 10, y-r s 10, '\right.$ 'k', 'linewidth', 1.2)

grid on

hold on

log $\log \left(x \_r s 5, y \_r s 5, '\right.$ 'k:', 'linewidth', 1.2)

응 $\log \log \left(x \_r s 3, y-r s 3, ' k\right.$ ', 'Iinewidth', 1.2)

$\log \log \left(x \_r s 2, y-r s 2, ' k-1\right.$, 'linewidth', 1.2)

loglog(x_ra, y-ra, 'k-.', 'linewidth', 1.2)

$\log \log (x r b 10, \operatorname{yrb} 10$, 'ko')

$\log \log (x r b 5, y r b 5, ' k \circ ')$

의 $\log \left(\mathrm{xrb3}, \mathrm{yrb3}\right.$, ' $\left.\mathrm{ko}^{\prime}\right)$

$\log \log (x r b 2, y r b 2$, 'ko')

$\log \log (x r b, y r b, '$ 'ko')

$\log \log \left(x \_r, y-r, \quad ' k-1\right)$

\% $\log \log (x r h t, y r h t, \quad ' k . ')$

$\operatorname{axis}\left(\left[\begin{array}{llll}2 & 60 & 1 e-4 & 5 e-2\end{array}\right]\right)$

号 legend('Point scatterer', 'Rayleigh scatterer', 'Smooth prolate spheroid', 'Rough pro: 


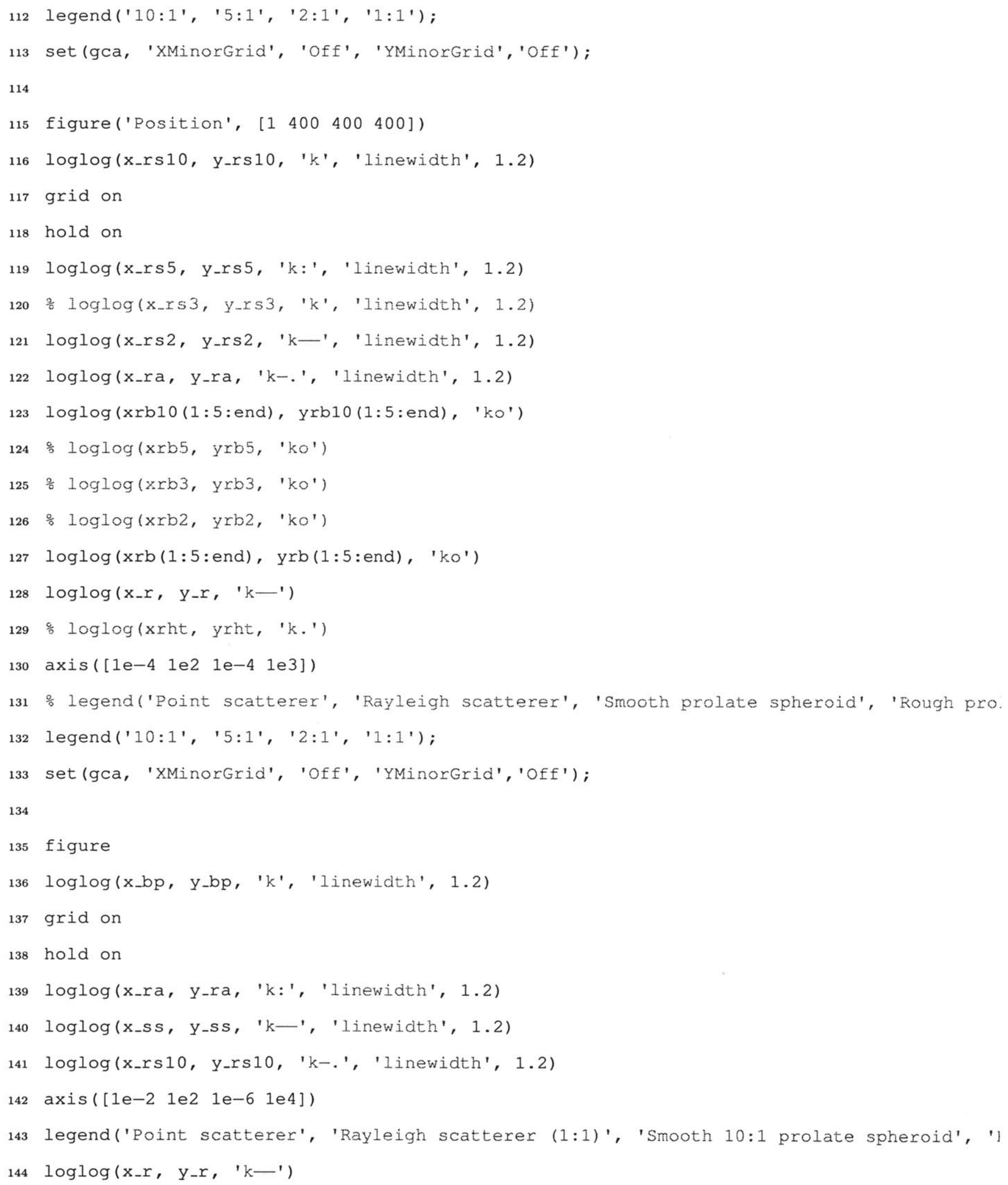




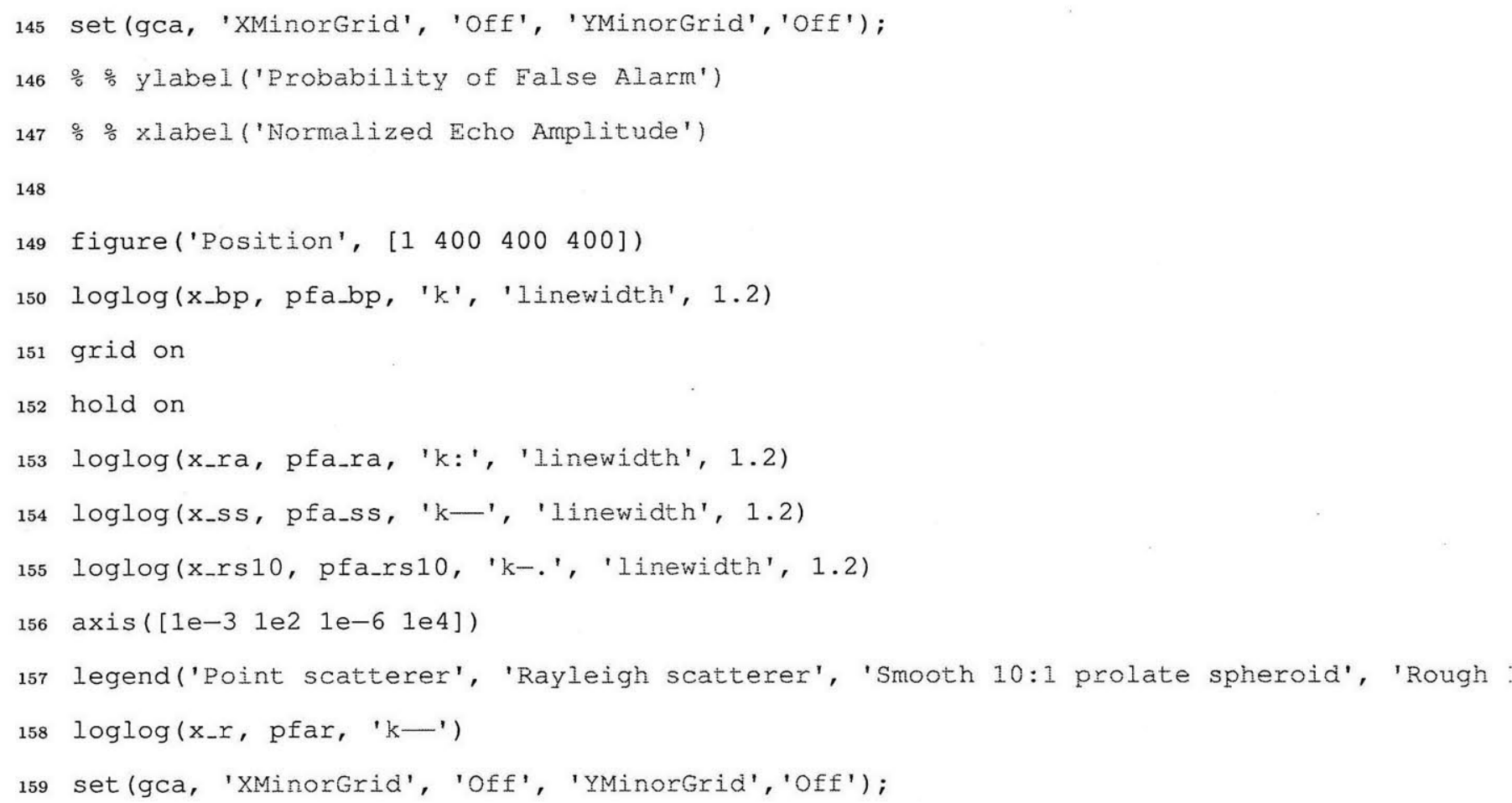




\section{Bibliography}

[1] D. A. Abraham and A. P. Lyons, Eds., Special issue on Non-Rayleigh reverberation and clutter, vol. 35, no. 2, IEEE J. Oceanic Eng., pp. 233-356, 2010.

[2] S. Watts and K. Ward, Eds., Special issue on Radar clutter, vol. 4, no. 2, IET Radar, Sonar and Navigation, pp. 143-328, 2010.

[3] E. Jakeman and K. D. Ridley, Modeling Fluctuations in Scattered Waves. Taylor and Francis, Boca Raton, FL, 2006.

[4] F. L. Chevalier, Principles of Radar and Sonar Signal Processing. Artech House Publishers, Norwood, MA, 2002.

[5] T. K. Stanton, D. Chu, and D. B. Reeder, "Non-rayleigh acoustic scattering characteristics of individual fish and zooplankton," IEEE J. Ocean. Eng., vol. 29, pp. 260-268, 2004.

[6] J. J. Bowman, T. B. A. Senior, and P. L. E. Uslenghi, Electromagnetic and acoustic scattering by simple shapes (Revised edition), Bowman, J. J., Senior, T. B. A., \& Uslenghi, P. L. E., Ed. Hemisphere Publishing Corp., New York, 1987.

[7] D. Chu and T. K. Stanton, "Statistics of echoes from a directional sonar beam insonifying finite numbers of single scatterers and patches of scatterers," IEEE J. Ocean Eng., vol. 35, pp. 267-277, 2010. 
[8] J. W. Goodman, Statistical Optics. Wiley, New York, 1985, ch. 1.

[9] T. K. Stanton and D. Chu, "Sound scattering by rough elongated elastic objects: II. fluctuations of scattered field," J. Acoust. Soc. Am., vol. 92, pp. 1665-1678, 1992.

[10] J. V. Gurley and T. K. Stanton, "Sound scattering by rough elongated elastic objects: III. experiment," J. Acoust. Soc. Am., vol. 94, pp. 2746-2755, 1993.

[11] A. Papoulis, Probability, random variables and stochastic processes, 4th ed. McGraw Hill, 2002.

[12] J. E. Ehrenberg, "A method for extracting the fish target strength distribution from acoustic echoes," in Proc. Conf. Eng. Ocean Environ, vol. 1, 1972, pp. 61-64.

[13] K. S. Trivedi, Probability and Statistics with Reliability, Queueing, and Computer Science Applications, 2nd ed. Wiley, New York, 2001.

[14] A. Papoulis, Probability and Statistics. Prentice Hall, 1989.

[15] V. K. Rohatgi, An Introduction to Probability Theory and Mathematical Statistics. Wiley, New York, 1976.

[16] A. T. de Hoop, Handbook of radiation and scattering of waves. Academic Press, London, 1995.

[17] L. E. Kinsler, A. R. Frey, A. B. Coppens, and J. V. Sanders, Fundamentals of Acoustics, 4th ed. Wiley, New York, 1999.

[18] A. D. Kotsis and J. A. Roumeliotis, "Electromagnetic scattering by a metallic spheroid using shape perturbation method," Progress in Electromagnetics Research, vol. 67, pp. 113-134, 2007. 
[19] R. D. Spence and S. Granger, "The scattering of sound from a prolate spheroid," J. Acoust. Soc. Am., vol. 23, no. 6, pp. 701-706, 1951.

[20] D. Chu, T. K. Stanton, and J. M. Jech, "Modeling of broadband backscattering by swimbladder bearing fish over a wide range of frequencies," in ICES SEAFACTS, Bergen, Norway, Jun. 2008.

[21] M. Born and E. Wolf, Principles of Optics. Pergamon, London, 1953.

[22] C. Eckart, "The scattering of sound from the sea surface," J. Acoust. Soc. Am., vol. 25 , no. 3 , pp. $566-570,1953$.

[23] D. A. Abraham, "The effect of multipath on the envelope statistics of bottom clutter," IEEE J. Ocean. Eng., vol. 32, no. 4, pp. 848-861, 2007. 